\title{
Geometry of Real Forms of the Complex Neumann System
}

Tina Novak

To cite this article: Tina Novak (2016) Geometry of Real Forms of the Complex Neumann System, Journal of Nonlinear Mathematical Physics 23:1, 74-91, DOI:

https://doi.org/10.1080/14029251.2016.1135643

To link to this article: https://doi.org/10.1080/14029251.2016.1135643

Published online: 04 January 2021 


\title{
Geometry of Real Forms of the Complex Neumann System
}

\author{
Tina Novak \\ Faculty of Mechanical Engineering, University of Ljubljana, Aškerčeva 6 \\ SI-1000 Ljubljana, Slovenia \\ tina.novak@fs.uni-lj.si
}

Received 30 May 2015

Accepted 23 October 2015

\begin{abstract}
In the paper, we study real forms of the complex generic Neumann system. We prove that the real forms are completely integrable Hamiltonian systems. The complex Neumann system is an example of the more general Mumford system. The Mumford system is characterized by the Lax pair $\left(L^{\mathbb{C}}(\lambda), M^{\mathbb{C}}(\lambda)\right)$ of $2 \times 2$ matrices, where $L^{\mathbb{C}}(\lambda)=\left[\begin{array}{ll}V^{\mathbb{C}}(\lambda) & W^{\mathbb{C}}(\lambda) \\ U^{\mathbb{C}}(\lambda) & -V^{\mathbb{C}}(\lambda)\end{array}\right]$ and $U^{\mathbb{C}}(\lambda), V^{\mathbb{C}}(\lambda), W^{\mathbb{C}}(\lambda)$ are suitable polynomials. The topology of a regular level set of the moment map of a real form is determined by the positions of the roots of the suitable real form of $U^{\mathbb{C}}(\lambda)$, with respect to the position of the values of suitable parameters of the system. For two families of the real forms of the complex Neumann system, we describe the topology of the regular level set of the moment map. For one of these two families the level sets are noncompact.

In the paper, we also give the formula which provides the relation between two systems of the first integrals in involution of the Neumann system. One of these systems is obtained from the Lax pair of the Mumford type, while the second is obtained from the Lax pair whose matrices are of dimension $(n+1) \times(n+1)$.
\end{abstract}

Keywords: integrable systems; Neumann system; Arnold-Liouville level sets; spectral curves; real structures; real forms.

2000 Mathematics Subject Classification: 37J35, 14H70, 70H06

\section{Introduction}

A Hamiltonian system $\left(M^{2 n}, \omega, H\right)$ on a symplectic manifold $\left(M^{2 n}, \omega\right)$ is completely integrable, if there exist $n$ functionally independent first integrals $H=H_{1}, H_{2}, \ldots, H_{n}$, which Poisson commute. The connected components of the level sets of regular values of the moment map $\widetilde{H}=\left(H_{1}, \ldots, H_{n}\right)$ are diffeomorphic to $T^{k} \times \mathbb{R}^{n-k}$. The solutions of the system are linearized on the level sets. This is the contents of the Arnold-Liouville theorem.

Let $(q, p) \in T^{*} \mathbb{R}^{n+1}$ be ambient coordinates and $A=\operatorname{diag}\left(a_{1}, \ldots, a_{n+1}\right)$. The C. Neumann system is a Hamiltonian system $\left(T^{*} S^{n}, \omega, H\right)$ on the cotangent bundle of the sphere where $\omega=\sum_{j=1}^{n+1} d q_{j} \wedge$ $d p_{j}$ and the Hamiltonian $H$ is

$$
H(q, p)=\frac{1}{2}\left(|q|^{2}|p|^{2}-\langle q, p\rangle^{2}\right)+\frac{1}{2}\left(2-|q|^{2}\right)\langle q, A q\rangle
$$

The Hamiltonian (1.1) is a modification of the Hamiltonian $H^{* *}(q, p)=\frac{1}{2}|p|^{2}+\frac{1}{2}\langle q, A q\rangle$ of the system of $n+1$ particles in the simple harmonic oscillation. This modification ensures that the particle stays in $T^{*} S^{n}$ and is well described in [3]. The Hamiltonian system of differential equations 
corresponding to the Neumann system is then $\dot{q}_{j}=\left\{q_{j}, H\right\}_{T^{*} S^{n}}$ and $\dot{p}_{j}=\left\{p_{j}, H\right\}_{T^{*} S^{n}}$, i.e

$$
\dot{q}=p, \quad \dot{p}=-A q-\left(|p|^{2}-\langle q, A q\rangle\right) q .
$$

The starting point of our study is a generic case of the Neumann system with spring constants $a_{1}<a_{2}<\ldots<a_{n+1}$. Such a system is completely Liouville integrable and it is a typical example of completely algebraically integrable systems (see [10], [12]). First integrals $F_{1}, \ldots, F_{n+1}$ are so-called Uhlenbeck's integrals (see [10], [13], [18]). If the constants $a_{1}, \ldots, a_{n+1}$ are positive, the Hamiltonian $H$ is a proper function and by the Arnold-Liouville theorem the connected components of the level set of the moment map $\widetilde{F}:(q, p) \rightarrow\left(F_{1}(q, p), \ldots, F_{n}(q, p)\right)$ for a regular value $c \in \mathbb{R}^{n}$ are $n$ dimensional tori $T^{n}$. If we omit the condition of the positivity of the spring constants, the Hamiltonian does not need to be proper. To prove that the connected components of the level sets are still tori and to count all tori in a fibre $\widetilde{F}^{-1}(c)$, we need some algebraic geometry.

C. Neumann system has the natural complexification $\left(T^{*}\left(S^{n}\right)^{\mathbb{C}}, \omega^{\mathbb{C}}, H^{\mathbb{C}}\right)$ and this is an example of the Mumford system (see [8], [10], [17]), so the equations of motion are equivalent to a Lax equation. In [10], it is proved that if the spectral curve $\mathscr{C}_{h}$ is smooth, the level set of the moment map (of the system's first integrals) is isomorphic to $\operatorname{Jac}\left(\mathscr{C}_{h}\right) \backslash \Theta$ where $\Theta$ is the theta divisor. Furthermore, it is shown that every connected component of the level set of the moment map for the real Neumann system is isomorphic to the torus $T^{n}$. Consequently, it is possible to count the number of connected components (see description in [4]).

We know that the complex quadric $\left(S^{n}\right)^{\mathbb{C}}$ has $n+1$ real structures parameterized by the signature. Because the spring constants are ordered, the real forms of the complex Neumann system with the same signature, but with different sequences of pluses and minuses, do not need to be equivalent. Therefore, we shall precisely denote all real forms of the Mumford system. If $\mathscr{S} \subseteq\{1,2, \ldots, n+1\}$, let $\varepsilon_{\mathscr{S}, j}=1$ if $j \in \mathscr{S}$ and $\varepsilon_{\mathscr{S}, j}=-1$ if $j \in \mathscr{S}^{c}$. We denote by $J_{\mathscr{S}}$ the diagonal matrix with 1 on positions in $\mathscr{S}$ and -1 elsewhere. Consider the antiholomorphic involutive automorphism:

$$
\tau_{\mathscr{S}}: T^{*} \mathbb{C}^{n+1} \longrightarrow T^{*} \mathbb{C}^{n+1}, \quad(Q, P) \longmapsto\left(J_{\mathscr{S}} \bar{Q}, J_{\mathscr{S}} \bar{P}\right) .
$$

The real form of the complex Neumann system regarding $\tau_{\mathscr{S}}$ is the Hamiltonian system written as $\left(T^{*} \mathscr{H}_{\mathscr{S}}^{n}, \omega_{\mathscr{S}}, H_{\mathscr{S}}\right)$. In the special cases, when $\mathscr{S}=\{1,2, \ldots, k\}$ or $\mathscr{S}$ is the singleton $\mathscr{S}=\{k\}$ for some $k \in\{1, \ldots, n+1\}$, we shall denote the new Hamiltonian systems by $\left(T^{*} \mathscr{H}_{k}^{n}, \omega_{k}, H_{k}\right)$ and $\left(T^{*} \mathscr{H}_{\{k\}}^{n}, \omega_{\{k\}}, H_{\{k\}}\right)$ respectively.

Our first aim in this paper is to prove that the real forms $\left(T^{*} \mathscr{H}_{\mathscr{S}}^{n}, \omega_{\mathscr{S}}, H_{\mathscr{S}}\right)$ of the complex Neumann system are completely integrable and write down their first integrals. Our second and principal aim is to describe the geometry of the level sets of the moment map of the new real Hamiltonian systems $\left(T^{*} \mathscr{H}_{k}^{n}, \omega_{k}, H_{k}\right)$ and $\left(T^{*} \mathscr{H}_{\{k\}}^{n}, \omega_{\{k\}}, H_{\{k\}}\right)$. We prove the following theorems:

Theorem 1.1. Connected components of the regular level set of the moment map of the Hamiltonian systems $\left(T^{*} \mathscr{H}_{k}^{n}, \omega_{k}, H_{k}\right)$ for $k=1,2, \ldots, n+1$ are tori $T^{n}=S^{1} \times \ldots \times S^{1}$.

Theorem 1.2. Connected components of the regular level set of the moment map of the Hamiltonian systems $\left(T^{*} \mathscr{H}_{\{k\}}^{n}, \omega_{\{k\}}, H_{\{k\}}\right)$ for $k=2, \ldots, n+1$ are non-compact sets $\mathbb{R} \times T^{n-1}=\mathbb{R} \times \underbrace{S^{1} \times \ldots \times S^{1}}_{(n-1)-\text {-imes }}$.

We study only these two families of the real forms because the topology of their level sets in both cases is independent of the position of the regular value in the bifurcation diagram of the moment 
map. Examples 2.1 and 5.1 show that for the other real forms special attention must be paid to the position of the regular value in the bifurcation diagrams and this requires further study.

The sphere $S^{n}$ is a case of a symmetric space. One can construct integrable systems, similar to the Neumann's system on more general symmetric spaces (see [2], [14], [15]). In these constructions the use of real structures is essential. In the future, one could study the topology of the ArnoldLiouville sets of these systems and their relatives. In a more speculative vein, one could study certain properties of the (generalized) Maxwell-Bloch equation, which can be seen as chains of Neumann systems (see [16]).

Through studying the level sets of the moment maps for the real forms of the complex Neumann system, new coordinates on equiaxed hyperboloids are obtained. We call them conical hyperboloidal coordinates and we define them in the next section. In section 3, we define complexification and real forms of constraint manifolds and constrained Hamiltonian systems. Furthermore, we find a family of complex analytic functions on $T^{*} \mathbb{C}^{n+1}$ whose real forms with respect to the involution $\tau_{k}$ are easily calculated. In section 4 , we write down the Lax equation of the real forms of complex Neumann system. We prove that these new systems are completely integrable. We give the formula of the relations between Uhlenbeck's integrals and the first integrals appearing in Ratiu's paper [12]. In section 5, we prove theorems 1.1 and 1.2.

\section{Elliptic spherical and conical hyperboloidal coordinates}

In this section we shall expand the definition of the elliptic spherical coordinates and define the conical hyperboloidal coordinates on equiaxed hyperboloids. The elliptic spherical coordinates on $S^{2}$ were used by Carl Neumann in [11] and they are represented as a limit case of elliptic coordinates in [1]. The general definition on $S^{n}$ is described in [4], [9].

Let $q_{1}, \ldots, q_{n+1}$ be coordinates in $\mathbb{R}^{n+1}$ and $a_{1}<a_{2}<\ldots<a_{n+1}$ real constants. Consider two polynomials

$$
A(\lambda)=\prod_{j=1}^{n+1}\left(\lambda-a_{j}\right) \quad \text { and } \quad U(\lambda)=A(\lambda) \sum_{j=1}^{n+1} \frac{q_{j}^{2}}{\lambda-a_{j}} .
$$

Let $\mathscr{S}=\{m, m+1, \ldots, k\} \subseteq\{1,2, \ldots, n+1\}$ for some $m \in\{1,2, \ldots, n+1\}$ and some $k \in\{m, m+$ $1, \ldots, n+1\}$. Further, let $\varepsilon_{\mathscr{S}, j}=1$ if $j \in \mathscr{S}$ and $\varepsilon_{\mathscr{S}, j}=-1$ if $j \in \mathscr{S}^{c}$ and define another polynomial

$$
U_{\mathscr{S}}(\lambda):=A(\lambda) \sum_{j=1}^{n+1} \varepsilon_{\mathscr{S}, j} \frac{q_{j}^{2}}{\lambda-a_{j}} .
$$

Definition 2.1. Conical hyperboloidal coordinates $u_{\mathscr{S}, 1}, \ldots, u_{\mathscr{S}, n}$ on the hyperboloid $\sum_{j=1}^{n+1} \varepsilon_{\mathscr{S}, j} q_{j}^{2}=1$ where $\prod_{j=1}^{n+1} q_{j} \neq 0$ are the solutions of the equation $\sum_{j=1}^{n+1} \varepsilon_{\mathscr{S}, j} \frac{q_{j}^{2}}{\lambda-a_{j}}=0$, i.e. the roots of the polyno$\operatorname{mial} U_{\mathscr{S}}(\lambda)$.

Remark 2.1. For $\mathscr{S}=\{1, \ldots, n+1\}$ we get the elliptic spherical coordinates $u_{1}, \ldots, u_{n}$, which are the roots of the polynomial $U(\lambda)$. It can be seen easily, that we have $a_{1}<u_{1}<a_{2}<u_{2}<\ldots<a_{n}<$ $u_{n}<a_{n+1}$.

That the conical hyperboloidal coordinates are well defined we have to prove the following proposition: 
Proposition 2.1. If $\mathscr{S}=\{m, m+1, \ldots, k\}$ for some $m \in\{1,2, \ldots, n+1\}$, some $k \in\{m, \ldots, n+1\}$ and $\sum_{j=1}^{n+1} \varepsilon_{\mathscr{S}, j} q_{j}^{2}=1$, the equation

$$
\sum_{j=1}^{n+1} \varepsilon_{\mathscr{S}, j} \frac{q_{j}^{2}}{\lambda-a_{j}}=0
$$

has $n$ real roots $u_{1}, \ldots, u_{n}$. In relation to the constants $a_{j}$, they are ordered in the following way:

1. if $m=1$, we have $\mathscr{S}=\{1, \ldots, k\}$ and

$$
a_{1}<u_{1}<\ldots<u_{k-1}<a_{k}<a_{k+1}<u_{k}<\ldots<a_{n}<u_{n-1}<a_{n+1}<u_{n} .
$$

2. if $m=2, \ldots, n$ and $k=m$, we have the singleton $\mathscr{S}=\{k\}$ for $k=2, \ldots, n$ and

$$
\begin{gathered}
u_{1}<a_{1}<u_{2}<a_{2}<\ldots<u_{k-1}<a_{k-1}<a_{k}<a_{k+1}<u_{k}<a_{k+2}<u_{k+1}<\ldots \\
\ldots<a_{n}<u_{n-1}<a_{n+1}<u_{n} .
\end{gathered}
$$

3. if $k=m=n+1$, we have $\mathscr{S}=\{n+1\}$ and

$$
u_{1}<a_{1}<u_{2}<a_{2}<\ldots<u_{n-1}<a_{n-1}<u_{n}<a_{n}<a_{n+1} .
$$

4. if $m=2, \ldots, n$ and $k=m+1, \ldots, n$ :

$$
\begin{aligned}
& u_{1}<a_{1}<\ldots<u_{m-1}<a_{m-1}<a_{m}<u_{m}<a_{m+1}<u_{m+1}<\ldots \\
& \quad \ldots<u_{k-1}<a_{k}<a_{k+1}<u_{k}<a_{k+2}<u_{k+1}<\ldots<u_{n-1}<a_{n+1}<u_{n} .
\end{aligned}
$$

5. if $m=2, \ldots, n$ and $k=n+1$ :

$$
u_{1}<a_{1}<\ldots<u_{m-1}<a_{m-1}<a_{m}<u_{m}<a_{m+1}<u_{m+1}<\ldots<u_{n-1}<a_{n}<u_{n}<a_{n+1} .
$$

Proof. We shall prove only item 4 . Other items can be proved similarly. The roots of the equation (2.1) are zeros of the polynomial

$$
U_{\{m, \ldots, k\}}(\lambda)=-\sum_{j=1}^{m-1} q_{j}^{2} \prod_{\substack{l=1 \\ l \neq j}}^{n+1}\left(\lambda-a_{l}\right)+\sum_{j=m}^{k} q_{j}^{2} \prod_{\substack{l=1 \\ l \neq j}}^{n+1}\left(\lambda-a_{l}\right)-\sum_{j=k+1}^{n+1} q_{j}^{2} \prod_{\substack{l=1 \\ l \neq j}}^{n+1}\left(\lambda-a_{l}\right) .
$$

If $m=2, \ldots, n$ and $k=m+1, \ldots, n$, we have

$$
\operatorname{sgn}\left(U_{\{m, \ldots, k\}}\left(a_{j}\right)\right)=\left\{\begin{array}{ll}
(-1)^{n-j-1} & ; \text { if } j=m, \ldots, k \\
(-1)^{n-j} & ; \text { if } j=1, \ldots, m-1 \text { or } j=k+1, \ldots, n+1
\end{array} .\right.
$$

There are $m-2$ real roots of $U_{\{m, \ldots, k\}}$ in $\left(a_{1}, a_{m-1}\right)$, within $\left(a_{m}, a_{k}\right)$ there are $k-m$ real roots and $n-k$ real roots are in $\left(a_{k+1}, a_{n+1}\right)$. We have $n-2$ real roots in $\left(a_{1}, a_{n}\right)$. Since $\operatorname{sgn}\left(U_{\{m, . ., k\}}\left(a_{n+1}\right)\right)=$ -1 for the monic polynomial $U_{\{m, ., k\}}$ there exists another root in $\left(a_{n+1}, \infty\right)$. From (2.4), $\operatorname{sgn}\left(U_{\{m, \ldots, k\}}\left(a_{1}\right)\right)=(-1)^{n-1}$. But $U_{\{m, \ldots, k\}}$ is monic of degree $n$, hence $\operatorname{sgn}\left(U_{\{m, \ldots, k\}}(\lambda)\right)=(-1)^{n}$ if $\lambda<<0$. Therefore, we have another (and the last one) root in $\left(-\infty, a_{1}\right)$.

The meromorphic function $\sum_{j=1}^{n+1} \varepsilon_{\mathscr{S}, j} \frac{q_{j}^{2}}{\lambda-a_{j}}=\frac{U_{\mathscr{S}}(\lambda)}{A(\lambda)}=\frac{\prod_{j=1}^{n}\left(\lambda-u_{j}\right)}{\prod_{j=1}^{n+1}\left(\lambda-a_{j}\right)}$ of variable $\lambda$ has simple poles at $a_{j}$ and the coefficients $\varepsilon_{\mathscr{S}, j} q_{j}^{2}$ are the residues of $\frac{U_{\mathscr{S}}(\lambda)}{A(\lambda)}$. Next example shows that conical hyperboloidal coordinates do not exist everywhere for every $\mathscr{S}$. 
Example 2.1. Consider coordinates $\left(q_{1}, q_{2}, q_{3}\right) \in \mathbb{R}^{3}$ and the constants $a_{1}<a_{2}<a_{3}$. Take $\mathscr{S}=$ $\{1,3\}$. We have hyperboloid $q_{1}^{2}-q_{2}^{2}+q_{3}^{2}=1$ and

$$
U_{\{1,3\}}(\lambda)=\lambda^{2}+\left(-\left(a_{2}+a_{3}\right) q_{1}^{2}+\left(a_{1}+a_{3}\right)^{2} q_{2}^{2}-\left(a_{1}+a_{2}\right) q_{3}^{2}\right) \lambda+a_{2} a_{3} q_{1}^{2}-a_{1} a_{3} q_{2}^{2}+a_{1} a_{2} q_{3}^{2} .
$$

with discriminant $D=\left(\left(a_{1}-a_{3}\right)+\left(a_{1}-a_{2}\right) q_{1}^{2}+\left(a_{3}-a_{2}\right) q_{3}^{2}\right)^{2}-4\left(a_{1}-a_{2}\right)\left(a_{1}-a_{3}\right) q_{1}^{2}$. If we fix constants $a_{1}=1, a_{2}=2$ and $a_{3}=3$, the discriminant above at the point $\left(q_{1}, q_{2}, q_{3}\right)=(1,1,1)$ is negative but at $(1,3,3)$ is positive.

\section{Complexification and real forms}

We shall define Hamiltonian systems on hyperboloids as real forms of the complex Neumann system. We will outline the complexification of a Hamiltonian system as it is done in [5] and apply such a construction to a constraint system. Since in this section the order of spring constants $a_{1}, \ldots, a_{n+1}$ is not important, we shall mostly work with real forms corresponding to the antiholomorphic involutive automorphisms $\tau_{\mathscr{S}}$ (see (1.3) in Introduction) for $\mathscr{S}=\{1, \ldots, k\}$.

\subsection{Complexification and real forms in an ambient space.}

Likewise Gerdjikov et al. in [5], we begin with a real Hamiltonian system $\left\{T^{*} \mathbb{R}^{n+1}, \omega, H\right\}$, where $T^{*} \mathbb{R}^{n+1}$ has coordinates $(q, p)=\left(q_{1}, \ldots, q_{n+1}, p_{1}, \ldots, p_{n+1}\right), \omega=\sum_{j=1}^{n+1} d q_{j} \wedge d p_{j}$ and $H$ is Hamiltonian (usually real analytic function). Complexifying $\left\{T^{*} \mathbb{R}^{n+1}, \omega, H\right\}$, we get the system $\left\{T^{*} \mathbb{C}^{n+1}, \omega^{\mathbb{C}}, H^{\mathbb{C}}\right\}$. Real coordinates $(q, p)$ become complex coordinates $(Q, P)$ and we write $Q_{j}=q_{j, 0}+i q_{j, 1}$ and $P_{j}=p_{j, 0}+i p_{j, 1}$. The symplectic form is $\omega^{\mathbb{C}}=\sum_{j=1}^{n+1} d Q_{j} \wedge d P_{j}$ and $H^{\mathbb{C}}$ is the natural extension of $H$ to $T^{*} \mathbb{C}^{n+1}$. Denote by $H_{0}^{\mathbb{C}}$ and $H_{1}^{\mathbb{C}}$ the real part and the imaginary part of the complex function $H^{\mathbb{C}}$ and by $\omega_{0}^{\mathbb{C}}$ and $\omega_{1}^{\mathbb{C}}$ the real part and the imaginary part of the complex form $\omega^{\mathbb{C}}$. If the initial system $\left\{T^{*} \mathbb{R}^{n+1}, \omega, H\right\}$ is completely integrable, then the complexified Hamiltonian system $\left\{T^{*} \mathbb{C}^{n+1}, \omega^{\mathbb{C}}, H^{\mathbb{C}}\right\}$ is completely integrable in the complex. Consider the antiholomorphic involutive automorphism $\tau_{k}: \mathbb{C}^{n+1} \rightarrow \mathbb{C}^{n+1}$ defined by

$$
\tau_{k}(Q)=J_{k} \bar{Q} \quad \text { for } \quad J_{k}=\operatorname{diag}\{\underbrace{1, \ldots, 1}_{\mathrm{k}-\text { times }},-1, \ldots,-1\} .
$$

The space $\mathbb{R}_{k}^{n+1}:=\left\{\left(q_{1,0}, \ldots, q_{k, 0}, i q_{k+1,1}, \ldots, i q_{n+1,1}\right)\right\}$ is defined to be the fixed point set of the involutive automorphism $\tau_{k}$. We can lift the involution $\tau_{k}$ to the cotangent bundle $T^{*} \mathbb{C}^{n+1}$ in the natural way. Denote this lift by $\tau_{k}$ too. Fixed points of the lifted $\tau_{k}$ determine the set

$$
\begin{aligned}
T^{*} \mathbb{R}_{k}^{n+1} & =\left\{\left(q_{1,0}, \ldots, q_{k, 0}, i q_{k+1,1}, \ldots, i q_{n+1,1}, p_{1,0}, \ldots, p_{k, 0}, i p_{k+1,1}, \ldots, i p_{n+1,1}\right)\right\} \cong \\
& \cong\left\{\left(q_{1}, \ldots, q_{n+1}, p_{1}, \ldots, p_{n+1}\right)\right\} .
\end{aligned}
$$

The involution $\tau_{k}$ acts on functions by $\tau_{k}\left(f^{\mathbb{C}}(Q, P)\right):=f^{\mathbb{C}}\left(\tau_{k}(Q, P)\right)$. For a real analytic function $f$ and its complexification $f^{\mathbb{C}}$, the involution $\tau_{k}$ defines the real form $f_{k}(q, p)$ by

$$
f_{k}(q, p):=\left.\frac{1}{2}\left(f^{\mathbb{C}}(Q, P)+\tau_{k}\left(f^{\mathbb{C}}(Q, P)\right)\right)\right|_{T^{*} \mathbb{R}_{k}^{n+1}} .
$$

Lemma 3.1. If a polynomial $f: T^{*} \mathbb{R}^{n+1} \rightarrow \mathbb{R}$ in $2(n+1)$ variables $q_{1}, \ldots, q_{n+1}, p_{1}, \ldots, p_{n+1}$ contains only monomials $\prod_{j=1}^{n+1} q_{j}^{m_{j}} p_{j}^{r_{j}}$ where $m_{1}, \ldots, m_{n+1}, r_{1}, \ldots, r_{n+1}$ are even or $m_{j}$ is odd and $r_{j}$ is odd for 
some $j$, the real form of its complexification is simply $f_{k}(q, p)=\left.f_{0}^{\mathbb{C}}(Q, P)\right|_{T^{*} \mathbb{R}_{k}^{n+1}}=\left.f^{\mathbb{C}}(Q, P)\right|_{T^{*} \mathbb{R}_{k}^{n+1}}$.

Proof. It is enough to prove the lemma for the monomial $f(q, p)=\prod_{j=1}^{n+1} q_{j}^{m_{j}} p_{j}^{r_{j}}$. By the assumption, the number $m_{k+1}+\ldots+m_{n+1}+r_{k+1}+\ldots+r_{n+1}$ is even and we have

$$
\frac{1}{2}\left(f^{\mathbb{C}}(Q, P)+\tau_{k}\left(f^{\mathbb{C}}(Q, P)\right)\right)=\frac{1}{2}\left(\prod_{j=1}^{n+1} Q_{j}^{m_{j}} P_{j}^{r_{j}}+\overline{\prod_{j=1}^{n+1} Q_{j}^{m_{j}} P_{j}^{r_{j}}}\right)=f_{0}^{\mathbb{C}}(Q, P) .
$$

However, $Q_{j}^{2}=\left(q_{j, 0}^{2}-q_{j, 1}^{2}\right)+i 2 q_{j, 0} q_{j, 1}$, similarly $P_{j}^{2}=\left(p_{j, 0}^{2}-p_{j, 1}^{2}\right)+i 2 p_{j, 0} p_{j, 1}$ and $Q_{j} P_{j}=$ $\left(q_{j, 0} p_{j, 0}-q_{j, 1} p_{j, 1}\right)+i\left(q_{j, 0} p_{j, 1}+q_{j, 1} p_{j, 0}\right)$. From the assumption on exponents, every summand in the imaginary part of $\prod_{j=1}^{n+1} Q_{j}^{m_{j}} P_{j}^{r_{j}}$ contains at least one of the factors $q_{j, 0} q_{j, 1}, p_{j, 0} p_{j, 1}$ and $q_{j, 0} p_{j, 1}+q_{j, 1} p_{j, 0}$ for some $j$. But these factors vanish in the restriction to the $T^{*} \mathbb{R}_{k}^{n+1}$. Therefore, $\left.f_{1}^{\mathbb{C}}(Q, P)\right|_{T^{*} \mathbb{R}_{k}^{n+1}}=0$.

If $\langle q, \widetilde{q}\rangle=\sum_{j=1}^{n+1} q_{j} \widetilde{q}_{j}$ is the standard inner product in $\mathbb{R}^{n+1}$ defining the Euclidean structure on $\mathbb{R}^{n+1}$ and $|q|^{2}:=\langle q, q\rangle$ is the corresponding Euclidean metric, their natural complexifications are $\langle Q, \widetilde{Q}\rangle_{\mathbb{C}}=\sum_{j=1}^{n+1} Q_{j} \widetilde{Q}_{j}$ and $|Q|_{\mathbb{C}}^{2}=\langle Q, Q\rangle_{\mathbb{C}}$. The restriction of $\langle,\rangle_{\mathbb{C}}$ to $\mathbb{R}_{k}^{n+1}$ is $\langle q, \widetilde{q}\rangle_{k}=\sum_{j=1}^{n+1} \varepsilon_{j} q_{j} \widetilde{q}_{j}$ for $\varepsilon_{1}=\ldots=\varepsilon_{k}=1$ and $\varepsilon_{k+1}=\ldots=\varepsilon_{n+1}=-1$. This new product defines a pseudo-Euclidean structure on $\mathbb{R}_{k}^{n+1}$ of signature $(k, n+1-k)$. An indefinite pseudo-Euclidean metric is $|q|_{k}^{2}=\langle q, q\rangle_{k}$.

Example 3.1. For the real Hamiltonian $H(q, p)=\frac{1}{2}\left(|q|^{2}|p|^{2}-\langle q, p\rangle^{2}\right)+\frac{1}{2}\left(2-|q|^{2}\right)\langle q, A q\rangle$ of the Neumann system (see (1.1)), the complexified function is

$$
H^{\mathbb{C}}(Q, P)=\frac{1}{2}\left(|Q|_{\mathbb{C}}^{2}|P|_{\mathbb{C}}^{2}-\langle Q, P\rangle_{\mathbb{C}}^{2}\right)+\frac{1}{2}\left(2-|Q|_{\mathbb{C}}^{2}\right)\langle Q, A Q\rangle_{\mathbb{C}}
$$

The real form of $H^{\mathbb{C}}$ regarding $\tau_{k}$ is $H_{k}(q, p)=\frac{1}{2}\left(|q|_{k}^{2}|p|_{k}^{2}-\langle q, p\rangle_{k}^{2}\right)+\frac{1}{2}\left(2-|q|_{k}^{2}\right)\langle q, A q\rangle_{k}$.

Since the complex canonical symplectic 2-form $\omega^{\mathbb{C}}$ on the cotangent bundle $T^{*} \mathbb{C}^{n+1}$ is $\omega^{\mathbb{C}}=$ $\sum_{j=1}^{n+1} d Q_{j} \wedge d P_{j}$ its real form is $\omega_{k}=\sum_{j=1}^{n+1} \varepsilon_{j} d q_{j} \wedge d p_{j}$.

For the Poisson bracket $\{f, g\}=\omega\left(X_{f}, X_{g}\right)$ of two real analytic functions $f$ and $g$, its complexification is the Poisson bracket $\left\{f^{\mathbb{C}}, g^{\mathbb{C}}\right\}_{\mathbb{C}}=\sum_{j=1}^{n+1} \frac{\partial f^{\mathbb{C}}}{\partial Q_{j}} \frac{\partial g^{\mathbb{C}}}{\partial P_{j}}-\frac{\partial f^{\mathbb{C}}}{\partial P_{j}} \frac{\partial g^{\mathbb{C}}}{\partial Q_{j}}$. From the restriction to $T^{*} \mathbb{R}_{k}^{n+1}$, the real form of the complex Poisson bracket is $\left\{f_{k}, g_{k}\right\}_{k}=\sum_{j=1}^{n+1} \varepsilon_{j}\left(\frac{\partial f_{k}}{\partial q_{j}} \frac{\partial g_{k}}{\partial p_{j}}-\frac{\partial f_{k}}{\partial p_{j}} \frac{\partial g_{k}}{\partial q_{j}}\right)$. This bracket comes from the real form of the complexified symplectic form, i.e. $\left\{f_{k}, g_{k}\right\}_{k}=\omega_{k}\left(X_{f_{k}}, Y_{g_{k}}\right)$.

Proposition 3.1. If the initial Hamiltonian system $\left\{T^{*} \mathbb{R}^{n+1}, \omega, H\right\}$ is completely integrable, the real Hamiltonian form $\left\{T^{*} \mathbb{R}_{k}^{n+1}, \omega_{k}, H_{k}\right\}$ is completely integrable. If $H_{1}, \ldots, H_{n}$ are first integrals of the initial system, then

$$
H_{j, k}=\left.\frac{1}{2}\left(H_{j}^{\mathbb{C}}+\tau_{k}\left(H_{j}^{\mathbb{C}}\right)\right)\right|_{T^{*} \mathbb{R}_{k}^{n+1}} ; \quad j=1, \ldots, n
$$

are first integrals of the new Hamiltonian system.

The proof is in [5].

If $\mathscr{S}$ is an arbitrary subset of $\{1,2, \ldots, n+1\}$, the matrix $J_{\mathscr{S}}$ is a diagonal matrix with 1 on positions $\mathscr{S}$ and -1 on positions $\mathscr{S}^{\mathrm{c}}$. We define the antiholomorphic involutive automorphism $\tau_{\mathscr{S}}: \mathbb{C}^{n+1} \rightarrow \mathbb{C}^{n+1}$ by prescription $\tau_{\mathscr{S}}(Q)=J_{\mathscr{S}} \bar{Q}$. We shall denote its lift to $T^{*} \mathbb{C}^{n+1}$ by the same 
symbol. Fixed points sets of $\tau_{\mathscr{S}}$ are then $\mathbb{R}_{\mathscr{S}}^{n+1}$ and $T^{*} \mathbb{R}_{\mathscr{S}}^{n+1}$. A restriction of the product $\langle,\rangle_{\mathbb{C}}$ to $\mathbb{R}_{\mathscr{S}}^{n+1}$ is the product $\langle q, \widetilde{q}\rangle_{\mathscr{S}}:=\sum_{j=1}^{n+1} \varepsilon_{\mathscr{S}, j} q_{j} \widetilde{q}_{j}$ where $\varepsilon_{\mathscr{S}, j}=1$ if $j \in \mathscr{S}$ and $\varepsilon_{\mathscr{S}, j}=-1$ if $j \in \mathscr{S}^{\mathrm{c}}$. Analogically, we write for functions $f_{\mathscr{S}}=\left.\frac{1}{2}\left(f_{\mathbb{C}}+\tau_{\mathscr{S}}\left(f_{\mathbb{C}}\right)\right)\right|_{T^{*} \mathbb{R}_{\mathscr{S}}^{n+1}}$, 2-forms and Poisson brackets. Proposition 3.1 is also true for the real Hamiltonian form $\left\{T^{*} \mathbb{R}_{\mathscr{S}}^{n+1}, \omega_{\mathscr{S}}, H_{\mathscr{S}}\right\}$. First integrals of this system are functions $H_{j, \mathscr{S}}=\left.\frac{1}{2}\left(H_{j}^{\mathbb{C}}+\tau_{\mathscr{S}}\left(H_{j}^{\mathbb{C}}\right)\right)\right|_{T^{*} \mathbb{R}_{\mathscr{S}}^{n+1}}$ for $j=1, \ldots, n$.

\subsection{Complexification and real forms of constrained systems.}

Let us first consider the phase spaces. Let $\left\{\phi_{1}, \ldots, \phi_{m}\right\}$ be smooth functions on $\left(T^{*} \mathbb{R}^{n+1}, \omega\right)$ which define a map $\Phi: T^{*} \mathbb{R}^{n+1} \rightarrow \mathbb{R}^{m}$ by prescription $\Phi(q, p)=\left(\phi_{1}(q, p), \ldots, \phi_{m}(q, p)\right)$. In the case of the cotangent bundle $T^{*} \mathbb{R}^{n+1}$, we usually have an even number of constraint functions, $m=2 k$, where $\phi_{1}, \ldots, \phi_{k}$ are constraints on the base space $\mathbb{R}^{n+1}$ and $\phi_{k+1}=T^{*} \phi_{1}, \ldots, \phi_{2 k}=T^{*} \phi_{k}$ are obvious suitable derivatives. If $c \in \mathbb{R}^{m}$ is a regular value of $\Phi$, then the level set $\Phi^{-1}(c)$ is a smooth manifold named a constraint manifold. Functions $\phi_{j}$ are constraint functions. For the definition of constraint manifolds in an arbitrary symplectic manifold, see [3]. We say that $\Phi^{-1}(c)$ is a cosymplectic submanifold of $T^{*} \mathbb{R}^{n+1}$ if the matrix $\left(\left\{\phi_{j}, \phi_{l}\right\}(q, p)\right)$ of Poisson brackets of the constraints is invertible for every point $(q, p) \in \Phi^{-1}(c)$. We know that the cosymplectic submanifold $\Phi^{-1}(c)$ is a symplectic manifold for the symplectic form $\left.\omega\right|_{\Phi^{-1}(c)}$ (see [3]).

Let $\phi_{j}^{\mathbb{C}}=\phi_{j}^{\mathbb{C}}(Q, P)$ be a natural extension of $\phi_{j}$ to $T^{*} \mathbb{C}^{n+1}$. Then we get a complexified map $\Phi^{\mathbb{C}}: T^{*} \mathbb{C}^{n+1} \rightarrow \mathbb{C},(Q, P) \mapsto\left(\phi_{1}^{\mathbb{C}}(Q, P), \ldots, \phi_{m}^{\mathbb{C}}(Q, P)\right)$.

Definition 3.1. Complexification of the constraint manifold $\Phi^{-1}(c)$ is the naturally defined complex constraint manifold $\left(\Phi^{\mathbb{C}}\right)^{-1}(c)$.

Definition 3.2. The real form of the complex constraint manifold respect to the involution $\tau_{\mathscr{S}}: T^{*} \mathbb{C}^{n+1} \rightarrow T^{*} \mathbb{C}^{n+1}$ is the constraint manifold $\Phi_{\mathscr{S}}^{-1}(c)$ for the map $\Phi_{\mathscr{S}}(q, p)=$ $\left(\phi_{1, \mathscr{S}}(q, p), \ldots, \phi_{m, \mathscr{S}}(q, p)\right)$.

In the sequel we will study the following example.

Example 3.2. Let $T^{*} S^{n}=\left\{(q, p) \in \mathbb{R}^{n+1} \times \mathbb{R}^{n+1} ;|q|^{2}=1,\langle q, p\rangle=0\right\}$ be the cotangent bundle of the sphere $S^{n}$. Its complexification is the complex constraint manifold

$$
\left(T^{*} S^{n}\right)^{\mathbb{C}}=T^{*}\left(S^{n}\right)^{\mathbb{C}}=\left\{(Q, P) \in \mathbb{C}^{n+1} \times \mathbb{C}^{n+1} ;|Q|_{\mathbb{C}}^{2}=1 ;\langle Q, P\rangle_{\mathbb{C}}=0\right\} .
$$

The real form of (3.3) with respect to the involution $\tau_{\mathscr{S}}$ is the constraint manifold

$$
T^{*} \mathscr{H}_{\mathscr{S}}^{n}=\left\{(q, p) \in \mathbb{R}^{n+1} \times \mathbb{R}^{n+1} ;|q|_{\mathscr{S}}^{2}=1 \&\langle q, p\rangle_{\mathscr{S}}=0\right\},
$$

i.e. the cotangent bundle of the hyperboloid $\mathscr{H}_{\mathscr{S}}^{n}=\left\{q \in \mathbb{R}^{n+1} ;|q|_{\mathscr{S}}^{2}=1\right\}$.

Proposition 3.2. Let $\mathscr{S}$ be any subset of $\{1, \ldots, n+1\}$. The constraint manifold $T^{*} \mathscr{H}_{\mathscr{S}}^{n}$ (3.4) is a symplectic manifold for the symplectic form $\omega_{\mathscr{S}}=\sum_{j=1}^{n+1} \varepsilon_{\mathscr{S}, j} d q_{j} \wedge d p_{j}$.

Proof. We have to verify that the matrix of Poisson brackets of the constraints is invertible at every point $(q, p) \in T^{*} \mathscr{H}_{\mathscr{S}}^{n}$. Constraints for $T^{*} \mathscr{H}_{\mathscr{S}}^{n}$ are functions $\phi_{1, \mathscr{S}}(q, p)=-1+\sum_{j=1}^{n+1} \varepsilon_{\mathscr{S}, j} q_{j}^{2}$ and 
$\phi_{2, \mathscr{S}}(q, p)=\sum_{j=1}^{n+1} \varepsilon_{\mathscr{S}, j} q_{j} p_{j}$. Since

$$
\frac{\partial \phi_{1, \mathscr{S}}}{\partial q_{j}}(q, p)=2 \varepsilon_{\mathscr{S}, j} q_{j}, \frac{\partial \phi_{1, \mathscr{S}}}{\partial p_{j}}(q, p)=0, \frac{\partial \phi_{2, \mathscr{S}}}{\partial q_{j}}(q, p)=\boldsymbol{\varepsilon}_{\mathscr{S}, j} p_{j}, \frac{\partial \phi_{2, \mathscr{S}}}{\partial p_{j}}(q, p)=\boldsymbol{\varepsilon}_{\mathscr{S}, j} q_{j}
$$

and $\varepsilon_{\mathscr{S}, j}^{2}=1$ from the definition of the Poisson bracket is $\left\{\phi_{1, \mathscr{S}}, \phi_{2, \mathscr{S}}\right\} \mathscr{S}(q, p)=2$ for every $(q, p) \in$ $T^{*} \mathscr{H}_{\mathscr{S}}^{n}$. The matrix of Poisson brackets is therefore $\left(\left\{\phi_{j, \mathscr{S}}, \phi_{l, \mathscr{S}}\right\}\right)(q, p)=\left(\begin{array}{cc}0 & 2 \\ -2 & 0\end{array}\right)$.

We say that the Hamiltonian system is constrained if it is defined on a constraint symplectic submanifold $\left(\Phi^{-1}(c),\left.\omega\right|_{\Phi^{-1}(c)}\right)$ of a symplectic manifold $(M, \omega)$ and we write it in the form $\left\{\Phi^{-1}(c), \omega, H\right\}$. If $M=T^{*} \mathbb{R}^{n+1}$ and $(q, p) \in T^{*} \mathbb{R}^{n+1}$ are ambient coordinates, we write $H=H(q, p)$ and $\omega$ as a symplectic form on $T^{*} \mathbb{R}^{n+1}$. Complexification of the constrained Hamiltonian system is a complex constrained system $\left\{\left(\Phi^{\mathbb{C}}\right)^{-1}(c), \omega^{\mathbb{C}}, H^{\mathbb{C}}\right\}$

Definition 3.3. Let $\left(\Phi^{-1}(c), \omega, H\right)$ be a constrained Hamiltonian system in the ambient manifold $T^{*} \mathbb{R}^{n+1}$ for the canonical symplectic form $\omega=\sum_{j=1}^{n+1} d q_{j} \wedge d p_{j}$. A real form of the complex constrained Hamiltonian system $\left\{\left(\Phi^{\mathbb{C}}\right)^{-1}(c), \omega^{\mathbb{C}}, H^{\mathbb{C}}\right\}$ in respect to the involution $\tau_{\mathscr{S}}: T^{*} \mathbb{C}^{n+1} \rightarrow$ $T^{*} \mathbb{C}^{n+1}$ is the Hamiltonian system

$$
\left\{\Phi_{\mathscr{S}}^{-1}(c), \omega_{\mathscr{S}}, H_{\mathscr{S}}\right\} .
$$

Example 3.3. For the complex Neumann system $\left\{T^{*} S^{n \mathbb{C}}, \omega^{\mathbb{C}}, H^{\mathbb{C}}\right\}$, where the complex symplectic form is $\omega^{\mathbb{C}}=\sum_{j=1}^{n+1} d Q_{j} \wedge d P_{j}$ and $H^{\mathbb{C}}(Q, P)$ as in (3.1), the real form with respect to the involution $\tau_{\mathscr{S}}: T^{*} \mathbb{C}^{n} \rightarrow T^{*} \mathbb{C}^{n}$ is the Hamiltonian system $\left\{T^{*} \mathscr{H}_{\mathscr{S}}^{n}, \omega_{\mathscr{S}}, H_{\mathscr{S}}\right\}$. The symplectic form in ambient coordinates is $\omega_{\mathscr{S}}=\sum_{j=1}^{n+1} \varepsilon_{\mathscr{S}} d q_{j} \wedge d p_{j}$ and the Hamiltonian is $H_{\mathscr{S}}(q, p)=$ $\frac{1}{2}\left(|q|_{\mathscr{S}}^{2}|p|_{\mathscr{S}}^{2}-\langle q, p\rangle_{\mathscr{S}}^{2}\right)+\frac{1}{2}\left(2-|q|_{\mathscr{S}}^{2}\right)\langle q, A q\rangle_{\mathscr{S}}$

\section{Lax equation and integrability of $\left\{T^{*} \mathscr{H}_{\mathscr{S}}^{n}, \omega_{\mathscr{S}}, H_{\mathscr{S}}\right\}$}

The Lax equation $\frac{d}{d t} L(\lambda)=[M(\lambda), L(\lambda)]$ of the Hamiltonian system is an equation with elements $L(\lambda), M(\lambda)$ from loop algebra $\mathfrak{g}\left[\lambda, \lambda^{-1}\right]$ which is equivalent to the Hamiltonian system of differential equations of the motion. In many cases and also in the case of the Neumann system $L(\lambda)$ and $M(\lambda)$ are polynomials in real or complex variable $\lambda$ with coefficients in Lie algebra $\mathfrak{s l}(r, \mathbb{R})$ or $\mathfrak{s l}(r, \mathbb{C})$. For the Neumann system, we shall study the version of $r=2$.

We know that for the Neumann system $\left(T^{*} S^{n}, \omega, H\right)$ the Hamiltonian system of differential equations (1.2) is equivalent to the Lax equation $\frac{d}{d t} L(\lambda)=[M(\lambda), L(\lambda)]$ where $L(\lambda)=\left(\begin{array}{cc}V(\lambda) & W(\lambda) \\ U(\lambda) & -V(\lambda)\end{array}\right)$ for Jacobi's polynomials $U(\lambda), V(\lambda)$ and $W(\lambda)$ and $M(\lambda)=\left(\begin{array}{cc}0 & \lambda+|p|^{2} \\ -1 & 0\end{array}\right)$ (see [6], [10]).

For the complex Neumann system $\left(\left(T^{*} S^{n}\right)^{\mathbb{C}}, \omega^{\mathbb{C}}, H^{\mathbb{C}}\right)$ which is described in the previous section, the Hamiltonian system of differential equations remains of the same form, i.e.

$$
\dot{Q}=P, \quad \dot{P}=-A Q-\left(|P|_{\mathbb{C}}^{2}-\langle Q, A Q\rangle_{\mathbb{C}}\right) Q .
$$

This system is equivalent to the Lax equation

$$
\frac{d}{d t} L^{\mathbb{C}}(\lambda)=\left[M^{\mathbb{C}}(\lambda), L^{\mathbb{C}}(\lambda)\right]
$$


where $L^{\mathbb{C}}(\lambda)=\left(\begin{array}{cc}V^{\mathbb{C}}(\lambda) & W^{\mathbb{C}}(\lambda) \\ U^{\mathbb{C}}(\lambda) & -V^{\mathbb{C}}(\lambda)\end{array}\right)$ for Jacobi's polynomials with complex coefficients

$$
\begin{aligned}
U^{\mathbb{C}}(\lambda) & =A(\lambda) \sum_{j=1}^{n+1} \frac{Q_{j}^{2}}{\lambda-a_{j}}, \quad V^{\mathbb{C}}(\lambda)=A(\lambda) \sum_{j=1}^{n+1} \frac{-Q_{j} P_{j}}{\lambda-a_{j}} \quad \text { and } \\
W^{\mathbb{C}}(\lambda) & =A(\lambda) \sum_{j=1}^{n+1}\left(-1+\sum_{j=1}^{n+1} \frac{-P_{j}^{2}}{\lambda-a_{j}}\right)
\end{aligned}
$$

and $M^{\mathbb{C}}(\lambda)=\left(\begin{array}{cc}0 & \lambda+|P|_{\mathbb{C}}^{2} \\ -1 & 0\end{array}\right)$

Proposition 4.1. To the Hamiltonian system $\left\{T^{*} \mathscr{H}_{\mathscr{S}}^{n}, \omega_{\mathscr{S}}, H_{\mathscr{S}}\right\}$, which is a real form of the complex Neumann system $\left(T^{*} S^{n \mathbb{C}}, \omega^{\mathbb{C}}, H^{\mathbb{C}}\right\}$ with respect to the involution $\tau_{\mathscr{S}}: T^{*} \mathbb{C}^{n+1} \rightarrow T^{*} \mathbb{C}^{n+1}$, belong the Hamiltonian system of differential equations

$$
\dot{q}=p, \quad \dot{p}=-A q-\left(|p|_{\mathscr{S}}^{2}-\langle q, A q\rangle_{\mathscr{S}}\right) q
$$

and the Lax equation $\frac{d}{d t} L_{\mathscr{S}}(\lambda)=\left[M_{\mathscr{S}}(\lambda), L_{\mathscr{S}}(\lambda)\right]$ for the Lax pair

$$
L_{\mathscr{S}}(\lambda)=\left(\begin{array}{cc}
V_{\mathscr{S}}(\lambda) & W_{\mathscr{S}}(\lambda) \\
U_{\mathscr{S}}(\lambda) & -V_{\mathscr{S}}(\lambda)
\end{array}\right) \quad \text { and } \quad M_{\mathscr{S}}(\lambda)=\left(\begin{array}{cc}
0 & \lambda+|p|_{\mathscr{S}}^{2} \\
-1 & 0
\end{array}\right)
$$

Coefficients of the matrix $L_{\mathscr{S}}(\lambda)$ are polynomials

$$
\begin{aligned}
U_{\mathscr{S}}(\lambda) & =A(\lambda) \sum_{j=1}^{n+1} \varepsilon_{\mathscr{S}, j} \frac{q_{j}^{2}}{\lambda-a_{j}}, \quad V_{\mathscr{S}}(\lambda)=A(\lambda) \sum_{j=1}^{n+1} \varepsilon_{\mathscr{S}, j} \frac{-q_{j} p_{j}}{\lambda-a_{j}} \quad \text { and } \\
W_{\mathscr{S}}(\lambda) & =A(\lambda)\left(-1+\sum_{j=1}^{n+1} \varepsilon_{\mathscr{S}, j} \frac{-p_{j}^{2}}{\lambda-a_{j}}\right)
\end{aligned}
$$

where $\varepsilon_{\mathscr{S}, j}=1$ if $j \in \mathscr{S}$ and $\boldsymbol{\varepsilon}_{\mathscr{S}, j}=-1$ if $j \in\{1, \ldots, n+1\} \backslash \mathscr{S}$.

Remark 4.1. For $\mathscr{S}=\{1,2, \ldots, n+1\}$, we get the initial Neumann system with Jacobian's polynomial $U(\lambda), V(\lambda)$ and $W(\lambda)$, with real coefficients $\left(Q_{j}=q_{j}, P_{j}=p_{j}\right)$, mentioned above. In this case, we have $\varepsilon_{\mathscr{S}, j}=1$ for every $j$.

Proof. The proposition is independent of the order of $a_{1}, \ldots, a_{n+1}$. Therefore, it is enough to prove it for the subsets $\mathscr{S}=\{1, \ldots, k\}$ where $k=1, \ldots, n+1$. First, we prove that the system of differential equations (4.4) corresponds to the Hamiltonian system $\left\{T^{*} \mathscr{H}_{k}^{n}, \omega_{k}, H_{k}\right\}$. Remember that $\omega_{k}=\sum_{j=1}^{n+1} \varepsilon_{j} d q_{j} \wedge d p_{j}$ and $H_{k}(q, p)=\frac{1}{2}\left(|q|_{k}^{2}|p|_{k}^{2}-\langle q, p\rangle_{k}^{2}\right)+\frac{1}{2}\left(2-|q|_{k}^{2}\right)\langle q, A q\rangle_{k}$. For the system on $T^{*} \mathbb{R}_{k}^{n+1}$, it is enough to write down the equations $\dot{q}_{j}=\left\{q_{j}, H_{k}\right\}_{k^{*} \mathscr{H}_{k}^{n}}$ and $\dot{p}_{j}=\left\{p_{j}, H_{k}\right\}_{k_{T^{*}} \mathscr{H}_{k}^{n}}$ where the Poisson bracket is in the form $\{f, g\}_{k}=\sum_{j=1}^{n+1} \varepsilon_{j}\left(\frac{\partial f}{\partial q_{j}} \frac{\partial g}{\partial p_{j}}-\frac{\partial f}{\partial p_{j}} \frac{\partial g}{\partial q_{j}}\right)$. Direct calculation 
gives us

$$
\begin{aligned}
& \left\{q_{j}, H_{k}\right\}_{k}=|q|_{k}^{2} p_{j}-\langle q, p\rangle_{k} q_{j} \\
& \left\{p_{j}, H_{k}\right\}_{k}=-|p|_{k}^{2} q_{j}+\langle q, p\rangle_{k} p_{j}+\langle q, A q\rangle_{k} q_{j}-\left(2-|q|_{k}^{2}\right) a_{j} q_{j} .
\end{aligned}
$$

Therefore, on $T^{*} \mathscr{H}_{k}^{n}$, where $|q|_{k}^{2}=1$ and $\langle q, p\rangle_{k}=0$, the following applies

$$
\dot{q}_{j}=p_{j}, \quad \dot{p}_{j}=-a_{j} q_{j}-\left(|p|_{k}^{2}-\langle q, A q\rangle_{k}\right) q_{j}
$$

In $T^{*} \mathbb{C}^{n+1}$, we have complex coordinates $\left(Q_{1}, \ldots, Q_{n+1}, P_{1}, \ldots, P_{n+1}\right)$ where $Q_{j}=q_{j, 0}+i q_{j, 1}$ and $P_{j}=p_{j, 0}+i p_{j, 1}$. Fixed points of the involution $\tau_{k}: T^{*} \mathbb{C}^{n+1} \rightarrow T^{*} \mathbb{C}^{n+1}$ determine a subset in $T^{*} \mathbb{C}^{n+1}$ vanishing following real coordinates

$$
\begin{gathered}
q_{1,1}=\ldots=q_{k, 1}=q_{k+1,0}=\ldots=q_{n+1,0}=0 \\
p_{1,1}=\ldots=p_{k, 1}=p_{k+1,0}=\ldots=p_{n+1,0}=0 .
\end{gathered}
$$

Because the complex Hamiltonian system (4.1) and the complex Lax equation (4.2) are equivalent for all complex variables $Q_{1}, \ldots, Q_{n+1}, P_{1}, \ldots, P_{n+1}$, they are also equivalent if we impose condition (4.6). After the identification of coordinates

$$
\left\{\left(q_{1,0}, \ldots, q_{k, 0}, i q_{k+1,1}, \ldots, i q_{n+1,1}, p_{1,0}, \ldots, p_{k, 0}, i p_{k+1,1}, \ldots, i p_{n+1,1}\right)\right\} \cong\left\{\left(q_{1}, \ldots, q_{n+1}, p_{1}, \ldots, p_{n}\right\}\right.
$$

in $T^{*} \mathbb{R}_{k}^{n+1}$ the first line of the Hamiltonian system (4.1) is $\dot{q}=p$ and the second line becomes $\dot{p}=-A q-\left(|p|_{k}^{2}-\langle q, A q\rangle_{k}\right) q$.

Now we want to see what happens to the matrices in the Lax equation (4.2) after the restriction to $T^{*} \mathbb{R}_{k}^{n+1}$. The matrix $M^{\mathbb{C}}(\lambda)$ obviously becomes a matrix $M_{k}(\lambda)$ in the proposition. Since $\left.Q_{j}^{2}\right|_{T^{*} \mathbb{R}_{k}^{n+1}}=\varepsilon_{j} q_{j}^{2}$, similarly $\left.P_{j}^{2}\right|_{T^{*} \mathbb{R}_{k}^{n+1}}=\varepsilon_{j} p_{j}^{2}$ and $\left.Q_{j} P_{j}\right|_{T^{*} \mathbb{R}_{k}^{n+1}}=\varepsilon_{j} q_{j} p_{j}$, the restriction of matrix $L^{\mathbb{C}}(\lambda)$ to the cotangent bundle $T^{*} \mathbb{R}_{k}^{n+1}$ has a form mentioned in the proposition for polynomials $U_{k}(\lambda), V_{k}(\lambda)$ and $W_{k}(\lambda)$. Equivalence of the complex Lax equation and the complex Hamiltonian system of differential equations therefore implies equivalence of the Lax equation $\frac{d}{d t} L_{k}(\lambda)=$ $\left[M_{k}(\lambda), L_{k}(\lambda)\right]$ and the system of differential equations (4.4).

From Lax equation $\frac{d}{d t} L_{\mathscr{S}}(\lambda)=\left[M_{\mathscr{S}}(\lambda), L_{\mathscr{S}}(\lambda)\right]$, we can write the characteristic equation $\operatorname{det}\left(L_{\mathscr{S}}(\lambda)-\mu I\right)=0$ which defines the spectral curve for the real form of the complex Neumann system. Using a notation of the coefficients of the matrix $L_{\mathscr{S}}(\lambda)$ as in Proposition 4.1, the characteristic equation becomes $\mu^{2}=f_{\mathscr{S}}(\lambda)$ where $f_{\mathscr{S}}(\lambda):=U_{\mathscr{S}}(\lambda) W_{\mathscr{S}}(\lambda)+V_{\mathscr{S}}^{2}(\lambda)$. As done in [10], we can reorder the polynomial $f_{\mathscr{S}}(\lambda)$ to get conserved quantities of the system

$f_{\mathscr{S}}(\lambda)=A^{2}(\lambda)\left(\sum_{j=1}^{n+1} \varepsilon_{\mathscr{S}, j} \frac{q_{j}^{2}}{\lambda-a_{j}}\left(-1+\sum_{l=1}^{n+1} \varepsilon_{\mathscr{S}, l} \frac{-p_{l}^{2}}{\lambda-a_{l}}\right)+\left(\sum_{j=1}^{n+1} \varepsilon_{\mathscr{S}, j} \frac{-q_{j} p_{j}}{\lambda-a_{j}}\right)^{2}\right)=A^{2}(\lambda)\left(-\sum_{j=1}^{n+1} \varepsilon_{\mathscr{S}, j} \frac{G_{\mathscr{S}, j}}{\lambda-a_{j}}\right)$

for $G_{\mathscr{S}, j}:=q_{j}^{2}+\sum_{\substack{l=1 \\ l \neq j}}^{n+1} \varepsilon_{\mathscr{S}, l} \frac{K_{j, l}^{2}}{a_{j}-a_{l}}$ where $K_{j, l}=q_{j} p_{l}-q_{l} p_{j}$ 
Theorem 4.1. Functions

$$
G_{\mathscr{S}, j}(q, p)=q_{j}^{2}+\sum_{\substack{l=1 \\ l \neq j}}^{n+1} \varepsilon_{\mathscr{S}, l} \frac{K_{j, l}^{2}}{a_{j}-a_{l}} ; \quad j=1,2, \ldots, n+1
$$

are Poisson commuting first integrals for the Hamiltonian system $\left\{T^{*} \mathscr{H}_{\mathscr{S}}^{n}, \omega_{\mathscr{S}}, H_{\mathscr{S}}\right\}$. On the cotangent bundle $T^{*} \mathscr{H}_{\mathscr{S}}^{n}$, we have $\sum_{j=1}^{n+1} \varepsilon_{\mathscr{S}, j} G_{\mathscr{S}, j}=1$ and $\sum_{j=1}^{n+1} \varepsilon_{\mathscr{S}, j} a_{j} G_{\mathscr{S}, j}=2 H_{\mathscr{S}}$.

Proof. From Lemma 4.8 in [10], it is enough to prove that $\left\{G_{\mathscr{S}, j}, G_{\mathscr{S}, l}\right\} \mathscr{S}=0$ and $\left\{\phi_{1, \mathscr{S}}, G_{\mathscr{S}, j}\right\}_{\mathscr{S}}=$ 0 for the constraint $\phi_{1, \mathscr{S}}=|q|_{\mathscr{S}}^{2}-1$. We shall write $G_{\mathscr{S}, j}=q_{j}^{2}+\psi_{\mathscr{S}, j}$. First, we prove the second equality. A partial derivative of the function $G_{\mathscr{S}, j}$ with respect to $p_{l}$ is

$$
\frac{\partial G_{\mathscr{S}, j}}{\partial p_{l}}=\frac{\partial \psi_{\mathscr{S}, j}}{\partial p_{l}}=\left\{\begin{array}{c}
2 \varepsilon_{\mathscr{S}, l} \frac{K_{j, l} q_{j}}{a_{j}-a_{l}} \quad ; l \neq j \\
-2 \sum_{\substack{m=1 \\
m \neq j}}^{n+1} \varepsilon_{\mathscr{S}, m} \frac{K_{j, m} q_{m}}{a_{j}-a_{m}} ; l=j .
\end{array}\right.
$$

Using this equality, we get

$$
\left\{\phi_{1, \mathscr{S}}, G_{\mathscr{S}, j}\right\}_{\mathscr{S}}=2 \sum_{l=1}^{n+1} q_{l} \frac{\partial G_{\mathscr{S}, j}}{\partial p_{l}}=2\left(-2 q_{j} \sum_{\substack{m=1 \\ m \neq j}}^{n+1} \varepsilon_{\mathscr{S}, m} \frac{K_{j, m} q_{m}}{a_{j}-a_{m}}+\sum_{\substack{l=1 \\ l \neq j}}^{n+1} q_{l} 2 \varepsilon_{\mathscr{S}, l} \frac{K_{j, l} q_{j}}{a_{j}-a_{l}}\right)=0 .
$$

To calculate $\left\{G_{\mathscr{S}, j}, G_{, \mathscr{S}, l}\right\}_{\mathscr{S}}$, we have to calculate some auxiliary brackets. The following applies: $\left\{K_{j, l}, K_{k, m}\right\}_{\mathscr{S}}=0$ if $\{j, l\} \cap\{k, m\}=\emptyset$ and $\left\{K_{j, l}, K_{j, m}\right\}_{\mathscr{S}}=\varepsilon_{\mathscr{S}, j} K_{l, m}$. If $l \neq j$, we have

$$
K_{j, \alpha} K_{l, \beta}\left\{K_{j, \alpha}, K_{l, \beta}\right\} \mathscr{S}=\left\{\begin{array}{c}
\varepsilon_{\mathscr{S}, \alpha} K_{j, l} K_{j, \alpha} K_{l, \alpha} ; \alpha=\beta \\
-\varepsilon_{\mathscr{S}, j} K_{\alpha, l} K_{j, \alpha} K_{l, j} ; j=\beta \\
-\varepsilon_{\mathscr{S}, l} K_{j, \beta} K_{j, l} K_{l, \beta} ; l=\alpha
\end{array}\right.
$$

and therefore

$$
\begin{aligned}
& \left\{\psi_{\mathscr{S}, j}, \psi_{\mathscr{S}, l}\right\}_{\mathscr{S}}=4 \sum_{\substack{\alpha \neq j \\
\beta \neq l}} \frac{\varepsilon_{\mathscr{S}, \alpha}}{a_{j}-a_{\alpha}} \frac{\varepsilon_{\mathscr{S}, \beta}}{a_{l}-a_{\beta}} K_{j, \alpha} K_{l, \beta}\left\{K_{j, \alpha}, K_{l, \beta}\right\}_{\mathscr{S}}= \\
& =4 K_{j, l} \sum_{\substack{\alpha \neq j \\
\text { or } l}} \frac{\varepsilon_{\mathscr{S}, \alpha} \varepsilon_{\mathscr{S}, \alpha} \boldsymbol{\varepsilon}_{\mathscr{S}, \alpha}}{\left(a_{j}-a_{\alpha}\right)\left(a_{l}-a_{\alpha}\right)} K_{j, \alpha} K_{l, \alpha}-4 K_{l, j} \sum_{\alpha \neq j} \frac{\varepsilon_{\mathscr{S}, \alpha} \varepsilon_{\mathscr{S}, j} \boldsymbol{\varepsilon}_{\mathscr{S}, j}}{\left(a_{j}-a_{\alpha}\right)\left(a_{l}-a_{j}\right)} K_{\alpha, l} K_{j, \alpha}- \\
& -4 K_{j, l} \sum_{\beta \neq l} \frac{\boldsymbol{\varepsilon}_{\mathscr{S}, l} \boldsymbol{\varepsilon}_{\mathscr{S}, \beta} \boldsymbol{\varepsilon}_{\mathscr{S}, l}}{\left(a_{j}-a_{l}\right)\left(a_{l}-a_{\beta}\right)} K_{j, \beta} K_{l, \beta}= \\
& =4 K_{j, l} \sum_{\substack{\alpha \neq j \\
\text { or } l}} \varepsilon_{\mathscr{S}, l} \frac{a_{l}-a_{j}-\left(a_{l}-a_{\alpha}\right)+a_{j}-a_{\alpha}}{\left(a_{j}-a_{\alpha}\right)\left(a_{l}-a_{\alpha}\right)\left(a_{l}-a_{j}\right)} K_{j, \alpha} K_{l, \alpha}=0 .
\end{aligned}
$$

Using (4.8), we get

$$
\begin{aligned}
\left\{G_{\mathscr{S}, j}, G_{\mathscr{S}, l}\right\}_{\mathscr{S}} & =\left\{q_{j}^{2}, q_{l}^{2}\right\}_{\mathscr{S}}+\left\{q_{j}^{2}, \psi_{\mathscr{S}, l}\right\}_{\mathscr{S}}+\left\{\psi_{\mathscr{S}, j}, q_{l}^{2}\right\}_{\mathscr{S}}= \\
& =0+\boldsymbol{\varepsilon}_{\mathscr{S}, j} 2 q_{j} \frac{\partial \psi_{\mathscr{S}, l}}{\partial p_{j}}-\boldsymbol{\varepsilon}_{\mathscr{S}, l} 2 q_{l} \frac{\partial \psi_{\mathscr{S}, j}}{\partial p_{l}}= \\
& =\varepsilon_{\mathscr{S}, j} 2 q_{j} \boldsymbol{\varepsilon}_{\mathscr{S}, j} \frac{2 K_{l, j} q_{l}}{a_{l}-a_{j}}-\boldsymbol{\varepsilon}_{\mathscr{S}, l} \boldsymbol{\varepsilon}_{\mathscr{S}, l} \frac{2 K_{j, l} q_{j}}{a_{j}-a_{l}} 2 q_{l}=0 .
\end{aligned}
$$


On $T^{*} \mathscr{H}_{\mathscr{S}}^{n}$ we have $\sum_{j=1}^{n+1} \varepsilon_{\mathscr{S}, j} q_{j}^{2}=1$ and $\sum_{j=1}^{n+1} \varepsilon_{\mathscr{S}, j} q_{j} p_{j}=0$. Using this and making some meaningful dispositions in sums $\sum_{j=1}^{n+1} \varepsilon_{\mathscr{S}, j} G_{j}$ and $\sum_{j=1}^{n+1} \varepsilon_{\mathscr{S}, j} a_{j} G_{j}$, we can easily prove both equalities.

Let $G_{j}(q, p)$ be Uhlenbeck's integrals

$$
G_{j}(q, p):=G_{\{1, \ldots, n+1\}, j}=q_{j}^{2}+\sum_{\substack{l=1 \\ l \neq j}}^{n+1} \frac{K_{j, l}^{2}}{a_{j}-a_{l}}
$$

of the Neumann system on the sphere. Another system of integrals, constructed by T. Ratiu in [12] often appears in the literature. Below, we describe the relation between these two sets of integrals. Denote by $Q$ and $K$ the matrices $Q=\left(q_{j} q_{l}\right)$ and $K=\left(K_{j, l}\right)$. In Introduction we define $A=\operatorname{diag}\left(a_{1}, \ldots, a_{n+1}\right)$. According to [12], $n$ first integrals in involution are functions

$$
f_{k}(Q, K)=\frac{1}{2(k+1)} \operatorname{Tr}\left(-\sum_{i=0}^{k} A^{i} Q A^{k-i}+\sum_{\substack{i+r+s=k-1 \\ i, r, s \geq 0}} A^{i} K A^{r} K A^{s}\right) \quad \text { for } \quad k=1, \ldots, n .
$$

First, we write the integrals $f_{k}(Q, K)$ as functions $I_{k}$ of variables $(q, p)$. By multiplying matrices, we get $\left(A^{i} Q A^{k-i}\right)_{j j}=a_{j}^{k} q_{j}^{2}$ and $\operatorname{Tr}\left(-\sum_{i=0}^{k} A^{i} Q A^{k-i}\right)=-(k+1) \sum_{j=1}^{n+1} a_{j}^{k} q_{j}^{2}$. For the second sum in (4.10) we can easily calculate, that $\left(K A^{s}\right)_{j m}=a_{m}^{s} K_{j, m}$ and $\left(A^{i} K A^{r}\right)_{j m}=a_{m}^{r} a_{j}^{i} K_{j, m}$ and

$$
\left(A^{i} K A^{r} K A^{s}\right)_{j m}=\sum_{l=1}^{n+1}\left(A^{i} K A^{r}\right)_{j l} \cdot\left(K A^{s}\right)_{l m}=\sum_{l=1}^{n+1} a_{j}^{i} a_{l}^{r} a_{m}^{s} K_{j, l} K_{l, m}
$$

Therefore, we have

$$
\operatorname{Tr}\left(A^{i} K A^{r} K^{s}\right)=\sum_{j=1}^{n+1}\left(A^{i} K A^{r} K A^{s}\right)_{j j}=-\sum_{j=1}^{n+1} \sum_{l=1}^{n+1} a_{j}^{i+s} a_{l}^{r} K_{j, l}^{2}
$$

and we can write

$$
I_{k}(q, p)=f_{k}(Q(q, p), K(q, p))=-\frac{1}{2} \sum_{j=1}^{n+1} a_{j}^{k} q_{j}^{2}-\frac{1}{2(k+1)} \sum_{\substack{i+r+s=k-1 \\ i, r, s \geq 0}}\left(\sum_{j=1}^{n+1} \sum_{l=1}^{n+1} a_{j}^{i+s} a_{l}^{r} K_{j, l}^{2}\right)
$$

Proposition 4.2. Uhlenbeck's first integrals $G_{j}(q, p)$ and first integrals $I_{k}(q, p)$ are related by the equations

$$
-2 I_{k}(q, p)=\sum_{j=1}^{n+1} a_{j}^{k} G_{j}(q, p) \quad \text { for } \quad k=1, \ldots, n
$$

Proof. For $k=1$, we have $I_{1}(q, p)=-H(q, p)$ (see [12]). From Theorem 4.1 above, we know that $\sum_{j=1}^{n+1} a_{j} G_{j}=2 H$. 
We have to check the equations for $k>1$. From (4.11), we get

$$
\begin{aligned}
I_{k}(q, p) & =-\frac{1}{2}\left(\sum_{j=1}^{n+1} a_{j}^{k} q_{j}^{2}+\frac{1}{k+1} \sum_{j=1}^{n+1} \sum_{l=1}^{n+1} K_{j, l}^{2} \sum_{\substack{i+s=0 \\
i, s \geq 0}}^{k-1} a_{j}^{i+s} a_{l}^{k-1-(i+s)}\right)= \\
& =-\frac{1}{2}\left(\sum_{j=1}^{n+1} a_{j}^{k} q_{j}^{2}+\frac{1}{k+1} \sum_{j=1}^{n+1} \sum_{l>j} K_{j, l}^{2} \sum_{m=0}^{k-1}(k+1) a_{j}^{m} a_{l}^{k-1-m}\right)= \\
& =-\frac{1}{2}\left(\sum_{j=1}^{n+1} a_{j}^{k} q_{j}^{2}+\sum_{j=1}^{n+1} \sum_{l>j} K_{j, l}^{2} \sum_{m=0}^{k-1} a_{j}^{m} a_{l}^{k-1-m}\right) .
\end{aligned}
$$

The second equality above is true because of the symmetry on $a_{j}$ and $a_{l}$. For $k>1$, we can write $a_{j}^{k}-a_{l}^{k}=\left(a_{j}-a_{l}\right) \sum_{m=0}^{k-1} a_{j}^{m} a_{l}^{k-1-m}$. Therefore, the following is true

$$
\begin{aligned}
\sum_{j=1}^{n+1} a_{j}^{k} G_{j} & =\sum_{j=1}^{n+1} a_{j}^{k} q_{j}^{2}+\sum_{j=1}^{n+1} \sum_{\substack{l=1 \\
l \neq j}}^{n+1} \frac{a_{j}^{k} K_{j, l}^{2}}{a_{j}-a_{l}}=\sum_{j=1}^{n+1} a_{j}^{k} q_{j}^{2}+\sum_{j=1}^{n+1} \sum_{l>j} \frac{\left(a_{j}^{k}-a_{l}^{k}\right) K_{j, l}^{2}}{a_{j}-a_{l}}= \\
& =\sum_{j=1}^{n+1} a_{j}^{k} q_{j}^{2}+\sum_{j=1}^{n+1} \sum_{l>j} K_{j, l}^{2} \sum_{m=0}^{k-1} a_{j}^{m} a_{l}^{k-1-m} .
\end{aligned}
$$

\section{Level sets of the moment maps of $\left\{T^{*} \mathscr{H}_{k}^{n}, \omega_{k}, H_{k}\right\}$ and $\left\{T^{*} \mathscr{H}_{\{k\}}^{n}, \omega_{\{k\}}, H_{\{k\}}\right\}$}

The complex Neumann system is an example of the Mumford system. The Mumford system is a complex system given by the Lax equation $\frac{d}{d t} L^{\mathbb{C}}(\lambda)=\left[M^{\mathbb{C}}(\lambda), L^{\mathbb{C}}(\lambda)\right]$ (see [7], [8], [10]), where $L^{\mathbb{C}}(\lambda)=\left(\begin{array}{cc}V^{\mathbb{C}}(\lambda) & W^{\mathbb{C}}(\lambda) \\ -U^{\mathbb{C}}(\lambda) & V^{\mathbb{C}}(\lambda)\end{array}\right)$. Elements of matrix $L^{\mathbb{C}}(\lambda)$ are polynomials $U^{\mathbb{C}}(\lambda)=\lambda^{n}+\widetilde{u}_{1} \lambda^{n-1}+$ $\ldots+\widetilde{u}_{n}, V^{\mathbb{C}}(\lambda)=\widetilde{v}_{1} \lambda^{n-1}+\ldots+\widetilde{v}_{n}$ and $W^{\mathbb{C}}(\lambda)=\lambda^{n+1}+\widetilde{w}_{1} \lambda^{n}+\widetilde{w}_{2} \lambda^{n-1}+\ldots+\widetilde{w}_{n+1}$ with complex coefficients $\widetilde{u}_{j}, \widetilde{v}_{l}, \widetilde{w}_{m}$. We write briefly $\widetilde{u}=\left(\widetilde{u}_{1}, \ldots, \widetilde{u}_{n}\right), \widetilde{v}=\left(\widetilde{v}_{1}, \ldots, \widetilde{v}_{n}\right)$ and $\widetilde{w}=\left(\widetilde{w}_{1}, \ldots, \widetilde{w}_{n+1}\right)$ and $(\widetilde{u}, \widetilde{v}, \widetilde{w}) \in \mathbb{C}^{3 n+1}$. To the Lax equation above belongs a hyperelliptic spectral curve $\mathscr{C}$ defined by $\mu^{2}=f(\lambda)$, where $f(\lambda)=-\operatorname{det}(L(\lambda))=U^{\mathbb{C}}(\lambda) W^{\mathbb{C}}(\lambda)+\left(V^{\mathbb{C}}(\lambda)\right)^{2}$. The polynomial $f$ of the form of decreased exponents of $\lambda$ is

$$
f(\lambda)=\lambda^{2 n+1}+H_{1} \lambda^{2 n}+H_{2} \lambda^{2 n-1}+\ldots+H_{2 n} \lambda+H_{2 n+1},
$$

where the coefficients are functions $H_{j}=H_{j}(\widetilde{u}, \widetilde{v}, \widetilde{w})$ for $j=1, \ldots, 2 n+1$. These functions are conserved quantities of the system.

Consider the moment map $\Phi: \mathbb{C}^{3 n+1} \rightarrow \mathbb{C}^{2 n+1},(\widetilde{u}, \widetilde{v}, \widetilde{w}) \mapsto\left(H_{1}(\widetilde{u}, \widetilde{v}, \widetilde{w}), \ldots, H_{2 n+1}(\widetilde{u}, \widetilde{v}, \widetilde{w})\right)$. Let $h=\left(h_{1}, \ldots, h_{2 n+1}\right) \in \mathbb{C}^{2 n+1}$ be a value of the moment map $\Phi$ and denote by $\mathscr{C}_{h}$ a hyperelliptic curve, locally defined by $\mu^{2}=f_{h}(\lambda)$ where $f_{h}(\lambda)=\lambda^{2 n+1}+h_{1} \lambda^{2 n}+\ldots+h_{2 n} \lambda+h_{2 n+1}$.

Proposition 5.1. If the spectral curve $\mathscr{C}_{h}$ determined by the equation $\mu^{2}=f_{h}(\lambda)$ is smooth, the level set $\Phi^{-1}(h)$ is isomorphic to the $\operatorname{Jac}\left(\mathscr{C}_{h}\right) \backslash \Theta$.

The proof of the proposition is in [10]. If $u_{1}, \ldots, u_{n}$ are roots (they are variables!) of the polynomial $U^{\mathbb{C}}$, we can write $U^{\mathbb{C}}(\lambda)=\prod_{j=1}^{n}\left(\lambda-u_{j}\right)$. By the equation $f_{h}(\lambda)=U^{\mathbb{C}}(\lambda) W^{\mathbb{C}}(\lambda)+\left(V^{\mathbb{C}}(\lambda)\right)^{2}$, the value $V^{\mathbb{C}}\left(u_{j}\right)=v_{j}$ is determined by $V^{\mathbb{C}}\left(u_{j}\right)= \pm \sqrt{f_{h}\left(u_{j}\right)}$. If the roots of $U^{\mathbb{C}}$ are pairwise different, 


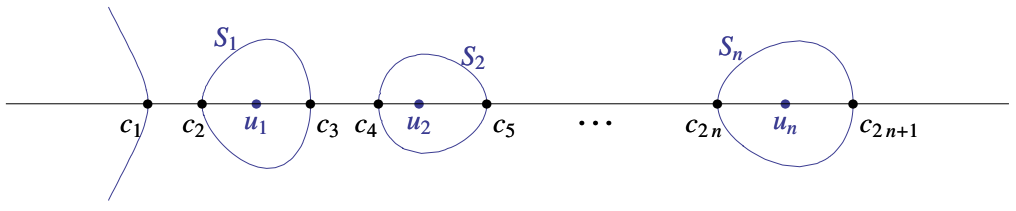

Fig. 1. Real part of the spectral curve $\mu^{2}=f(\lambda)$

polynomial $V^{\mathbb{C}}$ is an interpolation polynomial through the points $\left(u_{j}, v_{j}\right) \in \mathscr{C}_{h}$. The level set is therefore

$$
\begin{aligned}
\Phi^{-1}(h) & =\left\{(\widetilde{u}, \widetilde{v}, \widetilde{w}) \in \mathbb{C}^{3 n+1} ; U^{\mathbb{C}}(\lambda) W^{\mathbb{C}}(\lambda)+\left(V^{\mathbb{C}}(\lambda)\right)^{2}=f_{h}(\lambda)\right\} \cong \\
& \cong\left\{\left(u_{1}, \ldots, u_{n}, v_{1}, \ldots, v_{n}\right) \in \mathbb{C}^{2 n} ; U^{\mathbb{C}}(\lambda)=\prod_{j=1}^{n}\left(\lambda-u_{j}\right) \& v_{j}=V^{\mathbb{C}}\left(u_{j}\right) \text { for } j=1, \ldots, n\right\}
\end{aligned}
$$

From the Abel-Jacobi map, we know that $\operatorname{Jac}\left(\mathscr{C}_{h}\right) \backslash \Theta$ is isomorphic to the set of divisors $\operatorname{Div}_{+, 0}^{n}\left(\mathscr{C}_{h}\right) \cong\left\{D=\sum_{j=1}^{n} P_{j} ; P_{j} \neq \infty\right.$ for all $j$ and $P_{j} \neq \imath\left(P_{l}\right)$ if $\left.j \neq l\right\}$. Isomorphism between the level set $\Phi^{-1}(h)$ and $\operatorname{Div}_{+, 0}^{n}\left(\mathscr{C}_{h}\right)$ is the map $\left(u_{1}, \ldots, u_{n}, v_{1}, \ldots, v_{n}\right) \longmapsto \sum_{j=1}^{n} P_{j}$ where $P_{j}=\left(u_{j}, v_{j}\right) \in \mathscr{C}_{h}$.

In [10], it is proved that the (regular) level set of momentum map for the Neumann system is a $\mathbb{Z}_{2}^{n}$ covering of the real torus $T^{n}$ where $T^{n}$ is the set of real points of the affine part $\operatorname{Jac}\left(\mathscr{C}_{h}\right) \backslash \Theta$ of the Jacobian. To every point $(q, p) \in T^{*} S^{n}$, we could introduce the triple of polynomials $\pi:(q, p) \rightarrow$ $(U(\lambda), V(\lambda), W(\lambda)) \in \mathbb{C}^{3 n+1}$ by rule (4.5) taking $\varepsilon_{\mathscr{S}, j}=1$ for every $j \in\{1,2, \ldots, n+1\}$. There is a natural extension to the complexification $T^{*} S^{n} \mathbb{C}$, i.e. $\pi_{\mathbb{C}}:(Q, P) \rightarrow\left(U^{\mathbb{C}}(\lambda), V^{\mathbb{C}}(\lambda), W^{\mathbb{C}}(\lambda)\right)$ by rule (4.3). The map $\pi_{\mathbb{C}}$ is surjective and the preimage of the fixed value $\left(U^{\mathbb{C}}, V^{\mathbb{C}}, W^{\mathbb{C}}\right) \in \mathbb{C}^{3 n+1}$, where $U^{\mathbb{C}}, V^{\mathbb{C}}$ and $W^{\mathbb{C}}$ do not have a common root at $a_{k}$, is a subset

$$
\pi_{\mathbb{C}}^{-1}\left(U^{\mathbb{C}}, V^{\mathbb{C}}, W^{\mathbb{C}}\right)=\left\{\left(J_{\mathscr{S}} Q, J_{\mathscr{S}} P\right) ; U_{Q, P}^{\mathbb{C}}(\lambda), V_{Q, P}^{\mathbb{C}}(\lambda), W_{Q, P}^{\mathbb{C}}(\lambda) \text { as in }(4.3)\right\}
$$

where $J_{\mathscr{S}}$ is a diagonal matrix with 1 on positions $\mathscr{S}$ and -1 on positions $\{1,2, \ldots, n+1\} \backslash \mathscr{S}$ for any $\mathscr{S} \subseteq\{1,2, \ldots, n+1\}$. Next lemma gives us a condition on the spectral curve of the complex Neumann system for the coordinates $(Q, P)$ be real:

Lemma 5.1. Coordinates $Q$ and $P$ are real $\left(Q_{j}=q_{j}\right.$ and $\left.P_{j}=p_{j}\right)$ if and only if the coefficients of the polynomials $U^{\mathbb{C}}, V^{\mathbb{C}}$ and $W^{\mathbb{C}}$ are real and the roots $a_{1}, \ldots, a_{n+1}$ of $f_{h}(\lambda)=-A(\lambda) \prod_{j=1}^{n}\left(\lambda-b_{j}\right)$ and the roots $u_{1}, \ldots, u_{n}$ of $U^{\mathbb{C}}(\lambda)$ are arranged in the following order: $a_{1}<u_{1}<a_{2}<u_{2}<\ldots<$ $a_{n}<u_{n}<a_{n+1}$ and $b_{1} \in\left(-\infty, u_{1}\right)$ and $b_{j} \in\left(u_{j}, u_{j+1}\right)$ for $j=2, \ldots, n$.

The proof is in [10]. If we denote the ordered roots of the polynomial $f_{h}(\lambda)$ by $c_{1}, c_{2}, \ldots, c_{2 n+1}$, the roots of the polynomial $U^{\mathbb{C}}(\lambda)=U(\lambda)$ are on positions as in Figure 1. From the proof of Lemma above, we have $c_{2 n+1}=a_{n+1}$ and one member of the pair $\left(c_{2 j-1}, c_{2 j}\right)$ is $a_{j}$ and another one is $b_{j}$. From (5.1) and Lemma 5.1, the connected component of the regular level set of the moment map is the torus $S_{1} \times \ldots \times S_{n}$.

Proposition 5.2. Complex coordinates $Q$ and $P$ are equal to $\left(q_{1}, \ldots, q_{k}, i q_{k+1}, \ldots, i q_{n+1}\right)$ and $\left(p_{1}, \ldots, p_{k}, i p_{k+1}, \ldots, i p_{n+1}\right)$ respectively if and only if the coefficients of the polynomials $U^{\mathbb{C}}, V^{\mathbb{C}}$, and $W^{\mathbb{C}}$ are real and 
1. if $k=1$ the roots $a_{1}, \ldots, a_{n+1}$ of $f_{h}(\lambda)=-A(\lambda) B(\lambda)$ and the roots $u_{1,1}, \ldots, u_{1, n}$ of $U^{\mathbb{C}}(\lambda)$ are arranged in the following order

$$
\begin{aligned}
& a_{1}<a_{2}<u_{1,1}<a_{3}<u_{1,2}<a_{4}<\ldots<u_{1, n-2}<a_{n}<u_{1, n-1}<a_{n+1}<u_{1, n} \\
& \text { and } b_{j} \in\left(u_{1, j}, u_{1, j+1}\right) \text { for } j=1,2, \ldots, n-1 \text { and } b_{n} \in\left(u_{1, n}, \infty\right) .
\end{aligned}
$$

2. if $2 \leq k \leq n$ the roots $a_{1}, \ldots, a_{n+1}$ of $f_{h}(\lambda)$ and the roots $u_{k, 1}, \ldots, u_{k, n}$ of $U^{\mathbb{C}}(\lambda)$ are arranged in the following order

$$
\begin{aligned}
a_{1}<u_{k, 1}<a_{2}<u_{k, 2}<\ldots & <a_{k-1}<u_{k, k-1}<a_{k}<a_{k+1}<u_{k, k}<a_{k+2}<\ldots \\
\ldots & <u_{k, n-2}<a_{n}<u_{k, n-1}<a_{n+1}<u_{k, n}
\end{aligned}
$$

and $b_{j+1} \in\left(u_{k, j}, u_{k, j+1}\right)$ for $j=1, \ldots, k-2$ and $b_{j} \in\left(u_{k, j}, u_{k, j+1}\right)$ for $j=k, \ldots, n-1$ and $b_{1} \in\left(-\infty, u_{k, 1}\right)$ and $b_{n} \in\left(u_{k, n}, \infty\right)$.

Proof. We prove the case $k \geq 2$. For $k=1$ the proof goes quite similarly.

If $Q=\left(q_{1}, \ldots, q_{k}, i q_{k+1}, \ldots, i q_{n+1}\right)$ and $P=\left(p_{1}, \ldots, p_{k}, i p_{k+1}, \ldots, i p_{n+1}\right)$, polynomials $U^{\mathbb{C}}, V^{\mathbb{C}}$ and $W^{\mathbb{C}}$ are real and $U^{\mathbb{C}}(\lambda)=U_{k}(\lambda)=A(\lambda) \sum_{j=1}^{n+1} \varepsilon_{j} \frac{q_{j}^{2}}{\lambda-a_{j}}$ for $\varepsilon_{1}=\ldots=\varepsilon_{k}=1$ and $\varepsilon_{k+1}=\ldots=\varepsilon_{n+1}=-1$. The roots of $U_{k}$ are placed as mentioned in (2.2). From the property $f_{h}\left(u_{k, j}\right)>0$ there are $n-2$ roots $\left(\neq a_{j}\right)$ of $f_{h}: b_{j+1} \in\left(u_{k, j}, u_{k, j+1}\right)$ for $j=1,2, \ldots, k-2$ and $b_{j} \in\left(u_{k, j}, u_{k, j+1}\right)$ for $j=k, \ldots, n-1$. Because $f_{h}\left(u_{k, 1}\right)>0$ and $f_{h}(\lambda)>0$ for $\lambda<<0$, there must be another root $b_{1} \in\left(-\infty, u_{k, 1}\right)$. And $f_{h}\left(u_{k, n}\right)>0$ but $f_{h}(\lambda)<0$ if $\lambda>>0$. Therefore, another (and the last one) root is $b_{n} \in\left(u_{k, n}, \infty\right)$.

Conversely, by assumption, the polynomials $U^{\mathbb{C}}, V^{\mathbb{C}}$ and $W^{\mathbb{C}}$ are real and $U^{\mathbb{C}}$ and $f_{h}$ have roots in the mentioned order. Using a partial fractional decomposition, there is $U^{\mathbb{C}}(\lambda)=A(\lambda) \sum_{j=1}^{n+1} \frac{\alpha_{j}}{\lambda-a_{j}}$ or $U^{\mathbb{C}}(\lambda)=\sum_{j=1}^{n+1} \alpha_{j} \prod_{l \neq j}\left(\lambda-a_{l}\right)$ where $\alpha_{j}$ are real. Because $U^{\mathbb{C}}$ is monic of degree $n$, from the assumed positions of the roots $u_{k, j}$ is

$$
\operatorname{sgn}\left(U^{\mathbb{C}}\left(a_{j}\right)\right)=\left\{\begin{aligned}
(-1)^{n-j} & ; \text { if } j=k+1, \ldots, n+1 \\
(-1)^{n-j+1} & ; \text { if } j=1, \ldots, k
\end{aligned}\right.
$$

However, $U^{\mathbb{C}}\left(a_{j}\right)=\alpha_{j} \prod_{l \neq j}\left(a_{j}-a_{l}\right)$ and $\operatorname{sgn}\left(U^{\mathbb{C}}\left(a_{j}\right)\right)=\operatorname{sgn}\left(\alpha_{j}\right)(-1)^{n-j+1}$. By equating signs is $\operatorname{sgn}\left(\alpha_{j}\right)=1$ if $j=1, \ldots, k$ and the equations $Q_{j}^{2}=\alpha_{j}$ for $j=1, \ldots, k$ have only real roots. Respectively, $\operatorname{sgn}\left(\alpha_{j}\right)=-1$ if $j=k+1, \ldots, n+1$ and the equations $Q_{j}^{2}=\alpha_{j}$ for $j=k+1, \ldots, n+1$ have only imaginary roots. Similarly, we can prove that $P=\left(p_{1}, \ldots, p_{k}, i p_{k+1}, \ldots, i p_{n+1}\right)$ from the signs of the polynomial $W^{\mathbb{C}}$ at $a_{j}$.

Proof of Theorem 1. If we denote the ordered roots of $f_{h}(\lambda)$ by $c_{1}, c_{2}, \ldots, c_{2 n+1}$, the potions of the roots $u_{k, 1}=u_{1}, \ldots, u_{k, n}=u_{n}$ of the polynomial $U_{k}(\lambda)$ are the same as is presented in Figure 1. In this case the real curve in figure is the real part of the spectral curve in regard to the involution $\tau_{k}$. From isomorphism (5.1), Proposition 4.1 and proposition above, Theorem 1 is proved.

We finish our study with the similar proposition for another family of the real forms of the complex Neumann system. This family corresponds to the involution $\tau_{\mathscr{S}}$ where $\mathscr{S}=\{k\}$ for $k=$ $2,3, \ldots, n+1$. The case $\mathscr{S}=\{1\}$ is already included in the previous proposition. We shall write simply $u_{\{k\}, 1}=u_{1}, \ldots, u_{\{k\}, n}=u_{n}$.

Proposition 5.3. Complex coordinates $Q$ and $P$ are equal to

$\left(i q_{1}, \ldots, i q_{k-1}, q_{k}, i q_{k+1}, \ldots, i q_{n+1}\right)$ and $\left(i p_{1}, \ldots, i p_{k-1}, p_{k}, i p_{k+1}, \ldots, i p_{n+1}\right)$ respectively if and only if the coefficients of the polynomials $U^{\mathbb{C}}, V^{\mathbb{C}}$ and $W^{\mathbb{C}}$ are real and the roots of the polynomial $f_{h}(\lambda)$ and the roots $u_{1}, \ldots, u_{n}$ of $U^{\mathbb{C}}(\lambda)$ are arranged in the following order 
1. if $k=2, \ldots, n$ :

$$
\begin{gathered}
\qquad \begin{array}{c}
u_{1}<a_{1}<u_{2}<a_{2}<\ldots<u_{k-1}<a_{k-1}<a_{k}<a_{k+1}<u_{k}<a_{k+2}<u_{k+1}<\ldots \\
\ldots<a_{n}<u_{n-1}<a_{n+1}<u_{n} \\
\text { and } b_{j} \in\left(u_{j}, u_{j+1}\right) \text { for } j=1, \ldots, n-1 \text { and } b_{n} \in\left(u_{n}, \infty\right)
\end{array}
\end{gathered}
$$

2. if $k=n+1$ :

$$
\begin{aligned}
& \qquad u_{1}<a_{1}<u_{2}<a_{2}<\ldots<u_{n-1}<a_{n-1}<u_{n}<a_{n}<a_{n+1} \\
& \text { and } b_{j} \in\left(u_{j}, u_{j+1}\right) \text { for } j=1, \ldots, n-1 \text { and } b_{n} \in\left(u_{n}, \infty\right) \text {. }
\end{aligned}
$$

Proof. We prove the case $k=2, \ldots, n$. For $k=n+1$ the proof is similar.

If $Q=\left(i q_{1}, \ldots, i q_{k-1}, q_{k}, i q_{k+1}, \ldots, i q_{n+1}\right)$ and $P=\left(i p_{1}, \ldots, i p_{k-1}, p_{k}, i p_{k+1}, \ldots, i p_{n+1}\right)$ for $k=2, \ldots, n$, the polynomials $U^{\mathbb{C}}, V^{\mathbb{C}}$ and $W^{\mathbb{C}}$ are real and $U^{\mathbb{C}}(\lambda)=U_{\{k\}}(\lambda)=A(\lambda) \sum_{j=1}^{n+1} \varepsilon_{\{k\}, j} \frac{q_{j}^{2}}{\lambda-a_{j}}$ for $\varepsilon_{\{k\}, k}=$ 1 and $\varepsilon_{\{k\}, j}=-1$ if $j \neq k$. The zeros of $U_{\{k\}}$ are placed as mentioned in (2.3). Since $f_{h}(\lambda)$ has an odd number (one or three) of the roots noted by $a_{j}$ on the interval $\left(u_{j}, u_{j+1}\right)$ and $f_{h}\left(u_{j}\right)>0$, there exist another roots $b_{j} \in\left(u_{j}, u_{j+1}\right)$ for $j=1,2, \ldots, n-1$. Since $f_{h}\left(u_{1}\right)>0$ and $f_{h}(\lambda)<0$ for $\lambda>>0$, another (and the last one) root is $b_{n} \in\left(u_{n}, \infty\right)$.

Conversely, the polynomials $U^{\mathbb{C}}=U, V^{\mathbb{C}}=V$ and $W^{\mathbb{C}}=W$ are real and $U$ and $f_{h}$ have roots in the mentioned order. Using a partial fractional decomposition is $U(\lambda)=A(\lambda) \sum_{j=1}^{n+1} \frac{\alpha_{j}}{\lambda-a_{j}}$ or $U(\lambda)=$ $\sum_{j=1}^{n+1} \alpha_{j} \prod_{l \neq j}\left(\lambda-a_{l}\right)$ where $\alpha_{j}$ are real. Since $U$ is monic of degree $n$ and from the position of the roots depending on $a_{1}, \ldots, a_{n+1}$, the following applies:

$$
\operatorname{sgn}\left(U\left(a_{j}\right)\right)= \begin{cases}(-1)^{n-j} & ; \text { if } j=1, \ldots, k \text { and } j=k+1, \ldots, n+1 \\ (-1)^{n-j+1} ; \text { if } j=k .\end{cases}
$$

However, $U\left(a_{j}\right)=\alpha_{j} \prod_{l \neq j}\left(a_{j}-a_{l}\right)$ and $\operatorname{sgn}\left(U\left(a_{j}\right)\right)=\operatorname{sgn}\left(\alpha_{j}\right)(-1)^{n-j+1}$. Therefore, the equation $Q_{k}^{2}=\alpha_{k}$ has only real roots and the equations $Q_{j}^{2}=\alpha_{j}$ for $j=1, \ldots, k-1$ and $j=k+1, \ldots, n+1$ have only imaginary roots. Similarly, we could prove that $P=\left(i p_{1}, \ldots, i p_{k-1}, p_{k}, i p_{k+1}, \ldots, i p_{n+1}\right)$ from the signs of polynomial $W$ at $a_{j}$.

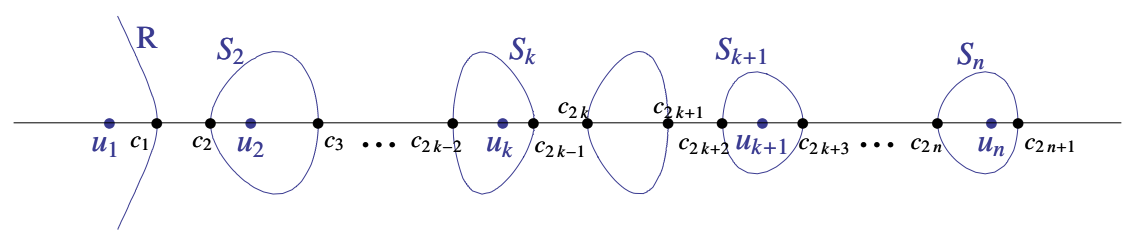

Fig. 2. Real part of the spectral curve $\mu^{2}=f(\lambda)$ in regard to the involution $\tau_{\{k\}}$

Proof of Theorem 2. If we denote the ordered roots of $f_{h}(\lambda)$ again by $c_{1}, c_{2}, \ldots, c_{2 n+1}$, the potions of the roots $u_{1}, . ., u_{n}$ of the polynomial $U_{\{k\}}(\lambda)$ are illustrated in Figure 2. The real curve in figure is the real part of the spectral curve respected to the involution $\tau_{\{k\}}$. From isomorphism (5.1), Proposition 4.1 and proposition above the connected components of the moment map's regular level set of $\left(T^{*} \mathscr{H}_{\{k\}}^{n}, \omega_{\{k\}}, H_{\{k\}}\right)$ for $k=2,3, \ldots, n+1$ is isomorphic to $\mathbb{R} \times T^{n-1} \cong R \times S_{2} \times \ldots \times S_{n}$. 
That the coordinates $\left(i q_{1}, \ldots, i q_{m-1}, q_{m}, \ldots, q_{k}, i q_{k+1}, \ldots, i q_{n+1}\right)$ and $\left(i p_{1}, \ldots, i p_{m-1}, p_{m}, \ldots, p_{k}, i p_{k+1}, \ldots, i p_{n+1}\right)$ for $m \geq 2$ and $k>m$ can not be included in the previous proposition and in Theorem 2 clearly explains the following example:

Example 5.1. For the hyperboloid $-q_{1}^{2}+q_{2}^{2}+q_{3}^{2}=1$, we have

$$
U_{\{2,3\}}(\lambda)=-q_{1}^{2}\left(\lambda-a_{2}\right)\left(\lambda-a_{3}\right)+q_{2}^{2}\left(\lambda-a_{1}\right)\left(\lambda-a_{3}\right)+q_{3}^{2}\left(\lambda-a_{1}\right)\left(\lambda-a_{2}\right) .
$$

The roots $u_{1}$ and $u_{2}$ of $U_{\{2,3\}}$ are placed: $u_{1}<a_{1}<a_{2}<u_{2}<a_{3}$ and $f_{h}\left(u_{1}\right) \geq 0$ and $f_{h}\left(u_{2}\right) \geq 0$. However, $f_{h}(\lambda)=-\left(\lambda-a_{1}\right)\left(\lambda-a_{2}\right)\left(\lambda-a_{3}\right)\left(\lambda-b_{1}\right)\left(\lambda-b_{2}\right)$ and the positions of the roots $b_{1}$ and $b_{2}$ are not determined precisely. There are three possibilities: $b_{1}, b_{2} \in\left(-\infty, u_{1}\right)$ or $b_{1}, b_{2} \in\left(u_{1}, u_{2}\right)$ or $b_{1}, b_{2} \in\left(u_{2}, \infty\right)$.

For the Hamiltonian system $\left(T^{*} \mathscr{H}_{\{2,3\}}^{2}, \omega_{\{2,3\}}, H_{\{2,3\}}\right)$, the topology of the level sets of the moment map depends on the position of the regular value of the moment map in the bifurcation diagram.

\section{Acknowledgments}

The author is grateful to advisor P. Saksida and to M. Vuk for all discussions and support during the study and the preparation of this paper. The author is also grateful to the referee for the careful reading, for the helpful suggestions and for turning the author's attention to the relation between two salient sets of integrals of the Neumann system.

\section{References}

[1] A.V. Bolsinov and A.T. Fomenko Integrable Hamiltonian Systems: Geometry, Topology, Classification (Chapman \& Hall/CRC, 2004)

[2] A.V. Bolsinov and B. Jovanovic, Magnetic Flows on Homogeneous Spaces, Comment. Math. Helv. 83 (2008) 679-700

[3] R.H. Cushman and L.M. Bates, Global Aspects of Classical Integrable Systems (Birkhäuser, 1997)

[4] H.R. Dullin, P.H. Richter, A.P. Veselov and H. Waalkens, Actions of the Neumann systems via PicardFuchs equaions, Phys. D 155 (2001) 159-183

[5] V.S. Gerdjikov, A. Kyuldjiev, G. Marmo and G. Vilasi, Real Hamiltonian forms of Hamiltonian systems, Eur. Phys. J. B 38 (2003) 635-649

[6] J. Harnad, Isospectral Flow and Liouville-Arnold Integration in Loop Algebras, Geometric and Quantum Aspects of Integrable Systems, Lecture Notes in Phys 424 (1993) 1-42

[7] R. Inoue, Y. Konishi and T. Yamazaki, Jacobian variety and integrable systems - after Mumford, Beauville and Vanhaecke, J. Geom. Phys. 57 (2007) 815-831

[8] R. Inoue, P. Vanhaecke and T. Yamazaki, Singular fiber of the Mumford system and rational soutions to the KdV hierarchy, Comm. Pure Appl. Math. 63 (2010) 508-532

[9] J. Moser, Various aspects of integrable Hamiltonian systems, Dynamical Systems (C.I.M.E. Summer Schools, Bressanone, 1978) Prog. Math. 8 (\& Birkhäuser, 1980) 233-289

[10] D. Mumford, Tata Lectures on Theta II, Progress in Mathematics 43 (Boston: Birkhäuser, 1984)

[11] C. Neumann, De problemate quodam mechanico, quod ad primam integralium ultraellipticorum classem revocatur, J. Reine Angew. Math. 56 (1859) 46-63

[12] T. Ratiu, The C. Neumann problem as a completely integrable system on an adjoint orbit, Trans. Amer. Math. Soc. 264 (1981) 321-329

[13] A.G. Reyman and M.A. Semenov-Tian-Shansky, Integrable systems II, Encyclopaedia Math. Sci. 16, Dynamical systems VII, ed V.I. Arnold and S.P. Novikov (Springer-Verlag Berlin Heidelberg 1994) 83-259

[14] P. Saksida, Nahm's equations and generalizations of the Neumann system, Proc. Lond. Math. Soc. 78 (1999) 701-720 
[15] P. Saksida, Integrable anharmonic oscillators on spheres and hyperbolic spaces, Nonlinearity 14 (2001) 977-994

[16] P. Saksida, Lattices of Neumann oscillators and Maxwell-Bloch equations, Nonlinearity 19 (2006) 747768

[17] P. Vanhaecke, Integrable systems in the realm of algebraic geometry, Lecture Notes in Mathematics 1638 (Springer-Verlag Berlin Heidelberg, 2001)

[18] M. Vuk, Algebraic integrability of confluent Neumann system, J. Phys. A 41(2008) 395201 